\title{
FINITE ELEMENT HETEROGENEOUS MULTISCALE METHODS WITH NEAR OPTIMAL COMPUTATIONAL COMPLEXITY*
}

\author{
ASSYR ABDULLE ${ }^{\dagger}$ AND BJORN ENGQUIST ${ }^{\ddagger}$
}

\begin{abstract}
This paper is concerned with a numerical method for multiscale elliptic problems. Using the framework of the heterogeneous multiscale methods (HMM), we propose a micro-macro approach which combines the finite element method (FEM) for the macroscopic solver and the pseudospectral method for the microsolver. Unlike the micro-macro methods based on the standard FEM proposed so far, in the HMM we obtain, for periodic homogenization problems, a method that has almost-linear complexity in the number of degrees of freedom of the discretization of the macro(slow) variable.
\end{abstract}

Key words. multiscale method, heterogeneous finite element method, spectral method, elliptic homogenization

AMS subject classifications. 65N30, 74Q05, 74Q15, 74Q20, 39A12

DOI. $10.1137 / 060676118$

1. Introduction. The numerical solution of problems encompassing a variety of strongly coupled scales poses major computational challenges in terms of analysis modeling and simulation. The direct numerical simulation of problems for which significant physical phenomena occur on length scales which differ by several orders of magnitude is often impossible, due to the computational cost for resolving the smallest scale.

For problems with scale separation, mathematical tools, such as homogenization theory, have been developed to derive "macro-" or "effective" or "homogenized" models (see [6], [21], [12] and the references therein). The numerical simulation of these macromodels can be done by standard methods. However, besides restrictive assumptions on the media, the simulation techniques based on these macromodels suffer from several drawbacks. The parameters of the effective models usually have to be computed numerically so that it is difficult to obtain an error control of the discretized macromodel. Furthermore, the small scale information is lost in these approaches. It can be recovered by solving corrector problems, but this procedure is as expensive as solving the full fine scale problem.

Introduced in [13], the heterogeneous multiscale method (HMM) has proved to be a useful framework for the design and analysis of multiscale methods. Such a method based on finite elements, the so-called finite element heterogeneous multiscale method (FE-HMM), has been developed in [2], [3], [4], [5], [14].

In these approaches, the unknown effective problem is solved directly with a coarse mesh by a macro FE solver. The unknown data of the macromodel are extracted on the fly by testing the microstructure on sampling domains with a micro FE method (FEM). Recently, a fully discrete error analysis has been derived for these types of methods [3], [4], [5]. The analysis in the aforementioned paper shows that for $N$

\footnotetext{
* Received by the editors November 28, 2006; accepted for publication (in revised form) June 7, 2007; published electronically December 21, 2007.

http://www.siam.org/journals/mms/6-4/67611.html

${ }^{\dagger}$ School of Mathematics and Maxwell Institute for Mathematical Sciences, University of Edinburgh, Edinburgh EH9 3JZ, Scotland (a.abdulle@ed.ac.uk).

${ }_{\ddagger}$ Mathematics Department, University of Texas at Austin, University Station C1200, Austin, TX 78712-0257 (engquist@ices.utexas.edu).
} 
"macro-" degrees of freedom, the overall complexity, taking into account the micro FE discretization, is superlinear in $N$. More precisely, consider piecewise linear FE space for the micro- and macroproblem and denote by $N_{m a c}$ and $N_{m i c}$ the degrees of freedom of the macro FEM and the micro FEM, respectively. Assume further that the costs (floating point operations) of the methods are proportional to their degrees of freedom (as, for example, when using a multigrid linear solver). Since the macro FEM has input data coming from the microproblems, the numerical error when solving these latter problems has an impact on the global error. For the approximation of the homogenized (upscaled) problem, the global error for solving numerically a multiscale elliptic problem with an HMM-type method is, as shown in [3] given by $e r r_{m a c}+e r r_{m i c}+e r r_{b}$, where $e r r_{m a c}$ is the error of the macro FEM, err mic is the error contribution from the micro FEM, and $\mathrm{err}_{b}$ comes from the (possibly inexact) boundary conditions and boundary layer terms [14]. We note that $\operatorname{err}_{b}=0$ with a heterogeneous multiscale-type method for the numerical approximation of periodic homogenization problems [2], [3]. When using a linear FEM for the microproblem, it has been shown in [3] that $e r r_{m i c}=\mathcal{O}\left(N_{m i c}^{-\frac{2}{d}}\right)$. Thus, for solving elliptic homogenization problems in the $L^{2}$ norm with the usual quadratic convergence rate we should use $N_{\text {mic }} \simeq N_{\text {mac }}$ and the total cost is $\mathcal{O}\left(N_{\text {mac }}^{2}\right)$, while in the $H^{1}$ norm (with a linear convergence rate) we can use $N_{m i c} \simeq N_{\text {mac }}^{1 / 2}$ and the total cost is $\mathcal{O}\left(N_{m a c}^{3 / 2}\right)$. Finally, if we want to approximate the fine scale solution, a reconstruction procedure has been proposed in [13], [1], and the analysis in [3] shows that the total cost (for the linear convergence rate in the $H^{1}$ norm) is $\mathcal{O}\left(N_{\text {mac }}^{2}\right)$. Notice that in this latter case, $\operatorname{err}_{b}=\sqrt{\varepsilon}$, where $\varepsilon$ is the length of the small scale oscillation.

In this paper, we propose and analyze a numerical method for elliptic multiscale problems based on a coupling of a macro FEM with a micropseudospectral method. Provided sufficient regularity of the conductivity tensor, we show that the microsolution has spectral accuracy. Furthermore, if the conductivity tensor is analytic, we show that the microsolution has exponential convergence. In this latter situation, the overall complexity is quasi-optimal, i.e., almost-linear, in the number of degrees of freedom $N_{m a c}$. More precisely, using the above notation, we show that err $_{m i c}$ decreases with a spectral or an exponential rate (these statements about spectral and exponential convergence rates will be made precise in the proofs).

For important classes of problems, including problems with periodic coefficients, with random stationary coefficients as well as for some nonlinear problems, the microproblems can be defined in periodic function spaces [13], [14], [2], [3], [4], [5]. The use of pseudospectral methods is thus well suited for these microproblems. Furthermore, using spectral methods on the microdomains (which can be chosen as squares/cubes) does not prevent us from applying the numerical method to domains with complicated geometry. It is the macrotriangulation which meshes the domain of the physical problem. Besides the optimal complexity for two scale problems, let us mention several issues in the HMM that can be addressed by the method proposed in this paper. An important issue in multiscale computation is high order methods. Theoretically, the FE-HMM can be easily constructed using several sampling domains within each macroelement [14]. However, the fully discrete analysis [3] shows that higher order microsolvers should also be implemented, for otherwise the computational complexity will be governed by $e r r_{m i c}$, the contribution of the low order microsolvers. By using spectral methods for the microsolver, it is possible to construct efficient high order FE-HMMs. Another issue is the numerical solution of problems with more than two (separated) scales. For such problems, the FE-HMM consists of a hierarchy of micro- 
macro methods. Refining simultaneously the whole hierarchy of meshes (based on the rate derived in [3], [4], [5]) can be computationally expensive. In this situation, almost-linear complexity with respect to the macrodegrees of freedom $N$ could also be achieved by coupling a macro FEM for the physical domain with pseudospectral methods for the hierarchy of microscales.

The paper is organized as follows. In section 2 we introduce the multiscale method based on the coupling of a macro FEM and micropseudospectral Fourier methods (FES-HMM); we state the main results and put them in perspective with previously obtained results. Section 3 is devoted to the analysis of the method and the proof of convergence results. In section 4 we extend our results for higher order macro FEMs. Finally, in section 5, we present numerical examples which illustrate the convergence rates of our method.

Notation. In what follows, $C>0$ denotes a generic constant, independent of $\varepsilon$, whose value can change at any occurrence but depends only on the quantities which are indicated explicitly. For $r=\left(r_{1}, \ldots, r_{d}\right) \in \mathbb{N}^{d}$, we denote $|r|=r_{1}+\cdots+r_{d}$, $D^{r}=\partial_{1}^{r_{1}} \ldots \partial_{d}^{r_{d}}$. We will consider the usual Sobolev space $H^{s}(\Omega)=\left\{u \in L^{2}(\Omega)\right.$; $\left.D^{r} u \in L^{2}(\Omega),|r| \leq s\right\}$, with norm $\|u\|_{H^{s}(\Omega)}=\left(\sum_{|r| \leq s}\left\|D^{r} u\right\|_{L^{2}(\Omega)}^{2}\right)^{1 / 2}$. We will also consider $H_{0}^{1}(\Omega)$ the closure of $C_{0}^{\infty}(\Omega)$ for the $\|\cdot\|_{H^{1}(\Omega)}$ norm and the spaces $W^{l, \infty}(\Omega)=\left\{u \in L^{\infty}(\Omega) ; D^{r} u \in L^{\infty}(\Omega),|r| \leq l\right\}$. We will also consider $W_{p e r}^{s}(Y)=$ $\left\{v \in H_{\text {per }}^{s}(Y) ; \int_{Y} v d x=0\right\}$, where $H_{\text {per }}^{s}(Y)$ is defined as the closure of $\mathcal{C}_{\text {per }}^{\infty}(Y)$ (the subset of $\mathcal{C}^{\infty}\left(\mathbb{R}^{d}\right)$ of periodic functions in the unit cube $\left.Y=(0,1)^{d}\right)$ for the $H^{s}$ norm. For $s=0$, we will denote $H_{p e r}^{0}(Y)=L_{p e r}^{2}(Y)$. Finally, we consider $C_{p e r}^{0}(Y)$, the set of continuous periodic complex-valued functions in $Y$.

2. Spectral heterogeneous multiscale FEM. In this section we first briefly recall homogenization theory and pseudospectral methods and introduce the multiscale FEM with microproblems solved by the pseudospectral Fourier method (FESHMM). At the end of the section we state the main convergence results. Although the analysis of our method will be performed in the periodic homogenization framework, we emphasize that the numerical method itself is not restricted to periodic homogenization problems.

2.1. Homogenization problems. We consider the following elliptic model problem in the domain $\Omega \subset \mathbb{R}^{d}$ :

$$
-\nabla \cdot\left(a^{\varepsilon} \nabla u^{\varepsilon}\right)=f \text { in } \Omega, \quad u^{\varepsilon}=0 \quad \text { on } \partial \Omega,
$$

where $\varepsilon$ is a small parameter that represents explicitly the multiscale nature (e.g., small scale) of the problem. Throughout, we will assume that the tensor $a^{\varepsilon}(x)$ is uniformly elliptic and bounded, i.e.,

$$
\gamma_{1}|\xi|^{2} \leq \xi^{T} a^{\varepsilon}(x) \xi \leq \gamma_{2}|\xi|^{2} \quad \forall \xi \in \mathbb{R}^{d} \text { and a.e. } x \in \Omega .
$$

For several classes of such multiscale problems, it is known from homogenization theory (see, e.g., [6, Chapter 1], [26]) that $u^{\varepsilon}$ converges (usually in a weak sense) to a "homogenized solution" $u^{0}$, solving an elliptic problem where the small scales have been averaged out.

The analysis of our methods will be presented for the case when the tensor $a^{\varepsilon}(x)=$ $a\left(x, \frac{x}{\varepsilon}\right)=a(x, y)$ is symmetric, coercive, and periodic with respect to each component of $y$ in the cube $Y=(0,1)^{d}, f \in L^{2}(\Omega), a_{i j}(x, \cdot) \in L^{\infty}\left(\mathbb{R}^{d}\right)$, and $x \rightarrow a_{i j}(x, \cdot)$ is the smooth from $\bar{\Omega} \rightarrow L^{\infty}\left(\mathbb{R}^{d}\right)$. In this situation, $u^{\varepsilon}$ converges weakly in $H_{0}^{1}(\Omega)$ to $u^{0}$, 
solution of the homogenized problem

$$
-\nabla \cdot\left(a^{0}(x) \nabla u^{0}\right)=f(x) \in \Omega, \quad u^{0}=0 \quad \text { on } \partial \Omega,
$$

where the homogenized diffusion coefficient $a^{0}$ is a smooth matrix with coefficients given by $a_{i j}^{0}(x)=\int_{Y}\left(a_{i j}(x, y)+\sum_{k=1}^{n} a_{i k}(x, y) \frac{\partial \chi^{j}}{\partial y_{k}}(x, y)\right) d y$. Here $\chi^{j}(x, \cdot)$ denote the solutions of the so-called cell problems

$$
\int_{Y} \nabla \chi^{j} a(x, y) \nabla v d y=\int_{Y}\left(a(x, y) e_{j}\right)^{T} \nabla v d y \quad \forall v \in W_{p e r}^{1}(Y), \quad j=1, \ldots, d,
$$

where $\left(e_{j}\right)_{j=1}^{d}$ is the canonical basis of $\mathbb{R}^{d}$. Classical homogenization theory [6], [26], [21] gives

$$
\left\|u^{\varepsilon}-u^{0}\right\|_{L^{2}(\Omega)} \leq C \varepsilon
$$

Some regularity on $\chi^{j}(x, \cdot)$ is needed for this estimate The assumption $\chi^{j}(x, \cdot) \in$ $W^{1, \infty}(Y)$, together with the assumption that the homogenized solution $u^{0}$ belongs to $H^{2}(\Omega)$, is sufficient for estimation (2.5) to hold (see [21, section 1.4], [18, Remark 3.3], $[17$, Remark 7]). In the nonperiodic case, there still exists a homogenized problem, but the homogenized matrix $a^{0}(x)$ is usually unknown [21]. We emphasize that our numerical method is not restricted to the periodic case (see the remark at the end of section 2.3 and section 5.3).

2.2. Spectral and pseudospectral Fourier approximation. We consider the Hilbert space $L^{2}(Y)$, the space of Lebesgue measurable square integrable functions $u: Y \longrightarrow \mathbb{C}$, where $Y=(0,1)^{d}$ with the scalar product $(u, v)=\int_{Y} u \bar{v} d y$ and the norm $\|u\|_{L^{2}(Y)}^{2}=(u, u)$.

Let us first suppose that $d=1$, and we set $I=(0,1)$. We recall that

$$
u \in L^{2}(I) \Longleftrightarrow u=\sum_{k=-\infty}^{k=\infty} \hat{u}_{k} e^{2 i k \pi y} \quad \text { with } \sum_{k=-\infty}^{k=\infty}\left|\hat{u}_{k}\right|^{2}<\infty .
$$

The coefficients $\hat{u}_{k}$ are given by $\hat{u}_{k}=\left(u, e^{2 i k \pi y}\right)$. We further consider for an integer $M>0$ the subspace spanned by $\operatorname{span}\left\{\psi_{k}=e^{2 i k \pi y} ;|k| \leq M\right\}$ and the $L^{2}$-orthogonal projection

$$
P_{M}(u)=\sum_{k=-M}^{k=M-1} \hat{u}_{k} \psi_{k} .
$$

In general it is not possible to calculate explicitly the Fourier coefficients $\hat{u}_{k}$ of the orthogonal projection. Let us define a mesh on $I$ with pseudospectral points given by

$$
I_{M}:=\left\{y_{l}=l h, l=0,1, \ldots, 2 M-1, h=1 / 2 M\right\} .
$$

We next consider the so-called pseudospectral method which is a collocation procedure at the pseudospectral points $y_{l}$ defined above. The discrete Fourier coefficients of a function $u \in C_{p e r}^{0}(I)$ with respect to the pseudospectral points $I_{M}$ are given by

$$
\tilde{u}_{k}=\frac{1}{2 M} \sum_{l=0}^{2 M-1} u\left(y_{l}\right) e^{-2 i k \pi y_{l}}
$$

Copyright $@$ by SIAM. Unauthorized reproduction of this article is prohibited. 
and the trigonometric interpolant of $u$ at the pseudospectral points $I_{M}$ is defined by

$$
u_{M}:=Q_{M}(u)=\sum_{k=-M}^{k=M-1} \tilde{u}_{k} \psi_{k} .
$$

Notice that $Q_{M}\left(u\left(y_{l}\right)\right)=u\left(y_{l}\right)$ for all $l=0,1, \ldots, 2 M-1$. In higher dimension $d>1$, we use the tensor product interpolant

$$
\mathbf{Q}_{M}(u)=\left(Q_{M}^{1} \otimes \cdots \otimes Q_{M}^{d}\right)(u),
$$

where $Q_{M}^{i}$ are given by (2.10). The $d$-dimensional pseudospectral mesh on $Y=(0,1)^{d}$ is defined as the tensor product of the 1-dimensional pseudospectral points

$$
Y_{M}:=\left\{\left(y_{l_{1}}, \ldots, y_{l_{d}}\right) ; y_{l_{i}} \in I_{M}\right\} .
$$

2.3. Multiscale FEM with pseudospectral microsolvers. We now construct the multiscale FEM with microproblems solved by the pseudospectral Fourier method. We concentrate here for simplicity on piecewise linear continuous FEMs in the macrospaces. We will consider higher order FEMs in section 4. We assume in what follows that the domain $\Omega$ where the multiscale problem is defined is a convex polygon. Therefore let the macro FE space be defined by

$$
S_{0}^{1}\left(\Omega, \mathcal{T}_{H}\right)=\left\{u^{H} \in H_{0}^{1}(\Omega) ;\left.u^{H}\right|_{K} \in \mathcal{P}^{1}(K) \forall K \in \mathcal{T}_{H}\right\},
$$

where $\mathcal{P}^{1}(K)$ is the space of linear polynomials on the triangle $K$, and $\mathcal{T}_{H}$ is a quasiuniform triangulation of $\Omega \subset \mathbb{R}^{d}$ of shape regular triangles $K$. By "macrofinite elements" we mean that $H$, the size of the triangulation, can be larger than the microlength scale $\varepsilon$.

REMARK 2.1. Standard a priori estimates for an $H^{2}$-regular solution $u^{\varepsilon}$ of problem (2.1) give $\left\|u^{\varepsilon}-u^{H}\right\|_{H^{1}(\Omega)} \leq C(H / \varepsilon)\|f\|_{L^{2}(\Omega)}$ (the factor $1 / \varepsilon$ is due to the small oscillations in $u^{\varepsilon}$; see [23]). The goal is thus to define a numerical method with a convergence rate independent of $\varepsilon$.

We consider for a macrotriangle $K \in \mathcal{T}_{h}$ a sampling subdomain centered at the barycenter $x_{K}$ of $K$ defined by $K_{\delta}=x_{K}+\delta[-1 / 2,1 / 2]^{d}$, where $\delta \geq \varepsilon$ (see the last paragraph of this section). We define a pseudospectral mesh on $K_{\delta}$ based on the mesh defined in (2.12):

$$
\begin{aligned}
K_{\delta M}: & =\left(x_{K}+\delta(-1 / 2,1 / 2)^{d}\right)+\delta Y_{M} \\
& =\left\{\xi_{l}=\left(\xi_{l_{1}}, \ldots, \xi_{l_{d}}\right), 0 \leq l_{i} \leq 2 M-1, i=1, \ldots, d\right\} .
\end{aligned}
$$

We also define

$$
S_{M}\left(K_{\delta}\right):=\operatorname{span}\left\{e^{2 i \pi k x / \varepsilon} ; x \in K_{\delta}, k \in \mathbb{Z}^{d},-M \leq k_{i} \leq M-1\right\} / \mathbb{R},
$$

where the quotient denotes the equivalence relation $u \simeq v \Longleftrightarrow u-v$ is a constant. We will also use $S_{M}(Y)$, which is defined as (2.15) for the domain $Y=(0,1)^{d}$ instead of $K_{\delta}$. We define a bilinear form on $S_{M}\left(K_{\delta}\right)$ built on the pseudospectral mesh $K_{\delta M}$ given by

$$
(u, v)_{M}:=\frac{\left|K_{\delta}\right|}{(2 M)^{d}} \sum_{l_{1}, \ldots, l_{d}=0}^{2 M-1} u\left(\xi_{l}\right) \bar{v}\left(\xi_{l}\right) .
$$

Copyright (C) by SIAM. Unauthorized reproduction of this article is prohibited. 
For functions $\mathbf{u}, \mathbf{v} \in S_{M}\left(K_{\delta}\right)$ we define

$$
(\mathbf{u}, \mathbf{v})_{M}:=\sum_{i=1}^{d}\left(u_{i}, v_{i}\right)_{M}
$$

We will use the same notation when working in the space $S_{M}(Y)$ with a pseudospectral mesh defined in (2.12) and a weight factor given by $\frac{|Y|}{(2 M)^{d}}=\frac{1}{(2 M)^{d}}$.

REMARK 2.2. Notice that

$$
\left(u_{M}, v_{M}\right)_{M}=\left(u_{M}, v_{M}\right) \quad \forall u_{M}, v_{M} \in S_{M}\left(K_{\varepsilon}\right) .
$$

This follows from the fact that the integration formula $\int_{0}^{1} v(x) d x \simeq \frac{1}{2 M} \sum_{j=0}^{2 M-1} v\left(x_{j}\right)$ is exact for $v \in S_{2 M}(I)$ (see formula (A.2)).

The FES-HMM for the elliptic homogenization problems, based on the macrospace $S_{0}^{1}\left(\Omega, \mathcal{T}_{H}\right)$, is defined by a modified macrobilinear form

$$
B\left(u^{H}, v^{H}\right)=\sum_{K \in \mathcal{T}_{H}} \frac{|K|}{\left|K_{\delta}\right|} \int_{K_{\delta}} \nabla u_{M} a\left(x_{K}, x / \varepsilon\right)\left(\nabla v_{M}\right)^{T} d x
$$

where $K_{\delta}=x_{K}+\delta[-1 / 2,1 / 2]^{d}$ is a sampling subdomain centered at the barycenter $x_{K}$ of $K$ and $|K|,\left|K_{\delta}\right|$ denote the measure of $K$ and $K_{\delta}$, respectively. The (unknown) microfunction $u_{M}$ is the solution of the following microproblem: for $u^{H} \in S_{0}^{1}\left(\Omega, \mathcal{T}_{H}\right)$, find $u_{M}$ such that $\left(u_{M}-u^{H}\right)=w_{M} \in S_{M}\left(K_{\delta}\right)$ and

$$
\left(a\left(x_{K}, x / \varepsilon\right) \nabla w_{M}, \nabla z_{M}\right)_{M}=\left(a\left(x_{K}, x / \varepsilon\right) \nabla u^{H}, \nabla z_{M}\right)_{M} \quad \forall z_{M} \in S_{M}\left(K_{\delta}\right) .
$$

The macro FES-HMM solution is defined by the following variational problem: find $u^{H} \in S_{0}^{1}\left(\Omega, \mathcal{T}_{H}\right)$ such that

$$
B\left(u^{H}, v^{H}\right)=\int_{\Omega} f v^{H} d x:=\left\langle f, v^{H}\right\rangle \quad \forall v^{H} \in S_{0}^{1}\left(\Omega, \mathcal{T}_{H}\right) .
$$

REMARK 2.3. Previous FE-HMMs are based on FEMs at the macro- and the microlevel [13], [14], [2], [3]. In these methods, the macrobilinear form is similar to (2.19), and the microfunctions are such that $\left(u^{h}-u^{H}\right) \in S_{\text {per }}^{1}\left(K_{\delta}, \mathcal{T}_{h}\right)$ and

$$
\int_{K_{\delta}} \nabla u^{h} a\left(x_{K}, x / \varepsilon\right)\left(\nabla z^{h}\right)^{T} d x=0 \quad \forall z^{h} \in S_{p e r}^{1}\left(K_{\delta}, \mathcal{T}_{h}\right),
$$

where

$$
S_{\text {per }}^{1}\left(K_{\delta}, \mathcal{T}_{h}\right)=\left\{z^{h} \in W_{p e r}^{1}\left(K_{\delta}\right) ;\left.z^{h}\right|_{T} \in \mathcal{P}^{1}(T), T \in \mathcal{T}_{h}\right\},
$$

and $\mathcal{P}^{1}(T)$ is the space of linear polynomials on the triangle $T$. Notice that setting $u^{h}-u^{H}=w^{h} \in S_{\text {per }}^{1}\left(K_{\delta}, \mathcal{I}_{h}\right)$, we can reformulate $(2.22)$ as a periodic problem with the right-hand side involving $u^{H}$ similarly as in (2.20).

Remark regarding the nonperiodic case. If the problem (2.1) is periodic with period $\varepsilon$, one can choose $\delta=\varepsilon$ in the above method. There are many practical multiscale problems with periodic structures but even more without periodicity or where the period is not known. Semidiscrete convergence but not computational complexity of the FE-HMM for these problems was studied in [14]. These problems 
typically require a computational microscale domain with a diameter $\delta$ that is larger than the period $\varepsilon$.

For problems with a periodic fast scale but with unknown period there are two error terms with implications for complexity: $\mathcal{O}(\delta)+\mathcal{O}(\varepsilon / \delta)$; see [14]. The first occurs if the coefficient is given as $a^{\varepsilon}$ and not with explicitly defined dependence on the periodic oscillatory component, and the second originates from a mismatch of the boundary conditions. The $\mathcal{O}(\delta)$ term can actually be improved to $\mathcal{O}\left(\delta^{q}\right)$ for any given integer $q$ by replacing the simple average used to approximate pointwise values in [14] by a weighted average. The weight or kernel should satisfy certain moment and regularity conditions; see Theorem 2.7 in [15]. The overall complexity can, anyway, not be bounded as well as with our periodic assumption. There are, however, cases for which only a narrow boundary layer is affected by the mismatch at the boundary of the microscale domain; see [19]. This is the reason why only a limited oversampling is often effective in the multiscale FEM. We can then have $\delta=\mathcal{O}(\varepsilon)$, and the overall complexity will be of the same order as in the known periodic case. Notice that in the case that the explicit form of the tensor $a^{\varepsilon}$ is not known or in the nonperiodic case, one should replace $S_{M}\left(K_{\delta}\right)$ defined in $(2.15)$ by a microspace based on Chebyshev pseudospectral points. One still retains the fast convergence of the microsolutions, provided sufficient regularity of the problem.

2.4. Main results. After showing that (2.21) is well-posed, we obtain, for periodic homogenization problems, the following convergence results, proved in section 3 and extended in section 4 for higher order macro FE spaces. Let $u^{0}$ be the solution of the homogenized problem (2.3), and assume $u^{0}$ is $H^{2}$ regular. Let $u^{H}$ be the solution of problem (2.21). Provided sufficient regularity of the conductivity tensor $a^{\varepsilon}(x, x / \varepsilon)$, we have the following theorem.

THEOREM 3.9.

$$
\left\|u^{0}-u^{H}\right\|_{H^{1}(\Omega)} \leq\left(C_{1} H+C_{2} M^{2(1-s)}\right),
$$

where $C_{2}$ depends on $\left\|a_{i j}^{\varepsilon}\right\|_{L^{\infty}\left(\Omega ; H_{p e r}^{s}(Y)\right)},\left\|\chi^{j}\right\|_{L^{\infty}\left(\Omega ; H_{p e r}^{s}(Y)\right)}$, where $H$ is the size of the triangulation of the macro FE space (2.13), and where $M^{d}$ is the number of pseudospectral points of the microspace $S_{M}\left(K_{\varepsilon}\right)$ given in (2.15).

COROllary.

$$
\begin{aligned}
& \left\|u^{0}-u^{H}\right\|_{L^{2}(\Omega)} \leq\left(C_{1} H^{2}+C_{2} M^{2(1-s)}\right)\|f\|_{L^{2}(\Omega)}, \\
& \left\|u^{\varepsilon}-u^{H}\right\|_{L^{2}(\Omega)} \leq\left(C_{1} H^{2}+C_{2} M^{2(1-s)}+C_{3} \varepsilon\right)\|f\|_{L^{2}(\Omega)} .
\end{aligned}
$$

Provided analycity of the conductivity tensor $a^{\varepsilon}(x, x / \varepsilon)$, we have the following theorem.

THEOREM 3.11.

$$
\left\|u^{0}-u^{H}\right\|_{H^{1}(\Omega)} \leq\left(C_{1} H+C_{2}\left(M(\log M)^{(d-1)} e^{-\alpha M}\right)^{2}\right),
$$

where $C_{2}$ depends on $\mathcal{B}$ (see Definition 3.4), where $H$ is the size of the triangulation of the macro FE space (2.13), and where $M^{d}$ is the number of pseudospectral points of the microspace $S_{M}\left(K_{\varepsilon}\right)$ given in $(2.15)$.

COROLlary.

$$
\begin{aligned}
& \left\|u^{0}-u^{H}\right\|_{L^{2}(\Omega)} \leq\left(C_{1} H^{2}+C_{2}\left(M(\log M)^{(d-1)} e^{-\alpha M}\right)^{2}\right)\|f\|_{L^{2}(\Omega)}, \\
& \left\|u^{\varepsilon}-u^{H}\right\|_{L^{2}(\Omega)} \leq\left(C_{1} H^{2}+C_{2}\left(M(\log M)^{(d-1)} e^{-\alpha M}\right)^{2}+C_{3} \varepsilon\right)\|f\|_{L^{2}(\Omega)} .
\end{aligned}
$$

Copyright (c) by SIAM. Unauthorized reproduction of this article is prohibited. 
Defining a numerical corrector $u_{p}^{\varepsilon}$ (see (3.31)) constrained by the known computed macrosolution $u^{H}$, we obtain an approximation of the fine scale solution $u^{\varepsilon}$. Provided sufficient regularity of the conductivity tensor $a^{\varepsilon}(x, x / \varepsilon)$, we have the following theorem.

THEOREM 3.13.

$$
\left\|u^{\varepsilon}-u_{p}^{\varepsilon}\right\|_{\bar{H}^{1}(\Omega)} \leq\left(C_{1} H+C_{2} M^{1-s}+C_{3} \sqrt{\varepsilon}\right),
$$

where $C_{2}$ depends on $\left\|a_{i j}^{\varepsilon}\right\|_{L^{\infty}\left(\Omega ; H_{p e r}^{s}(Y)\right)},\left\|\chi^{j}\right\|_{L^{\infty}\left(\Omega ; H_{p e r}^{s}(Y)\right)}$, where $H$ is the size of the triangulation of the macro FE space (2.13), and where $M^{d}$ is the number of pseudospectral points of the microspace $S_{M}\left(K_{\varepsilon}\right)$ given in (2.15).

The norm $\bar{H}^{1}(\Omega)$ (defined in (3.33)) is a broken Sobolev norm which is needed since the reconstructed solution $u_{p}^{\varepsilon}$ can be discontinuous across the macroelements $K$.

Provided analycity of the conductivity tensor $a^{\varepsilon}(x, x / \varepsilon)$, we have the following theorem.

THEOREM 3.14 .

$$
\left\|u^{\varepsilon}-u_{p}^{\varepsilon}\right\|_{\bar{H}^{1}(\Omega)} \leq\left(C_{1} H+C_{2} M(\log M)^{d-1} e^{-\alpha M}+C_{3} \sqrt{\varepsilon}\right),
$$

where $C_{2}$ depends on $\mathcal{B}$ (see Definition 3.4), where $H$ is the size of the triangulation of the macro FE space (2.13), and where $M^{d}$ is the number of pseudospectral points of the microspace $S_{M}\left(K_{\varepsilon}\right)$ given in (2.15).

Under the stated assumptions, we see in Theorems 3.11 and 3.14 and their corollaries that the contribution of the error of the microsolution, $M(\log M)^{d-1} e^{-\alpha M}$, decreases exponentially fast. Going back to the notation of the introduction and denoting by $N_{m a c}$ the degrees of freedom of the macro FEM, we see that for solving elliptic homogenization problems in the $L^{2}$ norm with the usual quadratic convergence rate we have a total cost of $\mathcal{O}\left(N_{\text {mac }}\right.$ ) (up to exponential convergence of the microproblem). The same is true for the $H^{1}$ norm and for the reconstructed solution. With the "standard" FE-HMM (see Remark 2.3) these costs are $\mathcal{O}\left(N_{\text {mac }}^{2}\right), \mathcal{O}\left(N_{\text {mac }}^{3 / 2}\right)$, $\mathcal{O}\left(N_{\text {mac }}^{2}\right)$, respectively [3], [5]. Thus, the proposed method achieves an almost-linear complexity (independent of $\varepsilon$ ) with respect to the number of macrodegrees of freedom.

Higher order macro FEMs. In section 4 we will extend the numerical method for conforming higher order macro FE spaces of polynomials of degree $p \geq 1$ on quasiuniform meshes, provided $u^{0}$ and $f$, the right-hand side of (2.3), are smooth enough ( $u^{0} \in H^{l+1}$ and $f \in H^{l}$ will suffice in what follows). For the original FE-HMM, using conforming FE spaces of polynomials of degree $p \geq 1$ for the macrosolver while keeping piecewise linear FE space for the microsolver one would obtain following [3] and [14]

$$
\left\|u^{0}-u^{H}\right\|_{H^{1}(\Omega)} \leq C\left(H^{\min (p, l)}+(h / \varepsilon)^{2}\right) .
$$

In the above estimate, $(h / \varepsilon)^{2}=\mathcal{O}\left(N_{m i c}^{-2 / d}\right)$ and $H^{s}=\mathcal{O}\left(N_{m a c}^{-s / d}\right)$, where $s=\min (p, l)$. Thus, not only is the complexity superlinear in the number of macrodegrees of freedom $N_{m a c}$, but for any $p, l \geq 1$ the global convergence rate cannot be faster than $\mathcal{O}\left(N_{m i c}^{-2 / d}\right)$ (quadratic rate) unless higher order FEMs are used for the microproblems. For the proposed approach, the same pseudospectral method, as defined in section 2.3, can be used for the microproblems with higher order macro FEMs. Provided sufficient regularity of the conductivity tensor $a^{\varepsilon}(x, x / \varepsilon)$, we obtain in section 4

$$
\left\|u^{0}-u^{H}\right\|_{H^{1}(\Omega)} \leq C_{1} H^{\min (p, l)}+C_{2} M^{2(1-s)} .
$$


Provided the analytic conductivity tensor we obtain in section 4 results of the form

$$
\left\|u^{0}-u^{H}\right\|_{H^{1}(\Omega)} \leq C_{1} H^{\min (p, l)}+C_{2}\left(M(\log M)^{d-1} e^{-\alpha M}\right)^{2},
$$

i.e., up to exponentially decreasing microerror, an overall almost-linear complexity of $\mathcal{O}\left(N_{\text {mac }}\right)$ for a macroconvergence rate of $H^{\min (p, l)}$. Convergence results for the $L^{2}$ norm will also be given for higher order macro FEMs in section 4.

3. Error analysis. We start by showing that the method is well-posed and then prove the main convergence results. In what follows, we set $\delta=\varepsilon$ in the FES-HMM defined in section 2.3 .

3.1. Well-posedness. We show in the following proposition that the bilinear form (2.19) is elliptic and bounded; thus (2.21) has a unique solution.

PROPOSITION 3.1. The problem (2.21) has a unique solution which satisfies

$$
\left\|u^{H}\right\|_{H^{1}(\Omega)} \leq C\|f\|_{L^{2}(\Omega)} .
$$

Proof. We first show that the problem (2.20) has a unique solution. The coercivity of the bilinear form defined in (2.20) follows from

$$
\begin{aligned}
& \left(a\left(x_{K}, x / \varepsilon\right) \nabla w_{M}, \nabla w_{M}^{T}\right)_{M}=\frac{\left|K_{\varepsilon}\right|}{(2 M)^{d}} \sum_{j=0}^{2 M-1} \nabla w_{M}\left(x_{j}\right) a\left(x_{K}, x_{j} / \varepsilon\right) \nabla w_{M}\left(x_{j}\right)^{T} \\
& \geq \gamma_{1} \frac{\left|K_{\varepsilon}\right|}{(2 M)^{d}} \sum_{j=0}^{2 M-1}\left|\nabla w_{M}\left(x_{j}\right)\right|^{2}=\gamma_{1} \int_{K_{\varepsilon}}\left|\nabla w_{M}(x)\right|^{2} d x=C\left\|w_{M}(x)\right\|_{H^{1}\left(K_{\varepsilon}\right)}^{2},
\end{aligned}
$$

where we used the coercivity of $a(x, x / \varepsilon)$ (see (2.2)), the equivalence of discrete and continuous scalar products for functions in $S_{M}\left(K_{\varepsilon}\right)$ (see Remark 2.2), and the norm equivalence $\left\|\nabla w_{M}(x)\right\|_{L^{2}\left(K_{\varepsilon}\right)} \simeq\left\|w_{M}(x)\right\|_{H^{1}\left(K_{\varepsilon}\right)}$. We next show that the bilinear form defined in $(2.20)$ is bounded:

$$
\begin{aligned}
& \left(a\left(x_{K}, x / \varepsilon\right) \nabla v_{M}, \nabla w_{M}^{T}\right)_{M} \\
& \quad \leq \gamma_{2}\left(\frac{\left|K_{\varepsilon}\right|}{(2 M)^{d}} \sum_{j=0}^{2 M-1} \nabla v_{M}\left(x_{j}\right)^{2}\right)^{1 / 2}\left(\frac{\left|K_{\varepsilon}\right|}{(2 M)^{d}} \sum_{j=0}^{2 M-1} \nabla w_{M}\left(x_{j}\right)^{2}\right)^{1 / 2} \\
& \leq \gamma_{2}\left\|\nabla v_{M}(x)\right\|_{L^{2}\left(K_{\varepsilon}\right)}\left\|\nabla w_{M}(x)\right\|_{L^{2}\left(K_{\varepsilon}\right)} \leq C\left\|v_{M}(x)\right\|_{H^{1}\left(K_{\varepsilon}\right)}\left\|w_{M}(x)\right\|_{H^{1}\left(K_{\varepsilon}\right)},
\end{aligned}
$$

where we used the boundedness of $a(x, x / \varepsilon)$, the Cauchy-Schwarz inequality, and, as above, the equivalence of discrete and continuous scalar products and norms. Thus, the existence and uniqueness of a solution of problem (2.20) follows from the LaxMilgram theorem.

It remains to show that (2.21) has a unique solution. Since $v_{M}-v^{H} \in S_{M}\left(K_{\varepsilon}\right) \subset$ $W_{\text {per }}^{1}\left(K_{\varepsilon}\right)$ and $\nabla v^{H}$ is constant over a macrotriangle $K$, we have

$$
\begin{aligned}
& \int_{K_{\varepsilon}}\left|\nabla v_{M}\right|^{2} d x=\int_{K_{\varepsilon}}\left|\nabla v_{M}-\nabla v^{H}\right|^{2} d x+\int_{K_{\varepsilon}}\left|\nabla v^{H}\right|^{2} d x, \\
& \int_{K_{\varepsilon}} \nabla v_{M} a\left(x_{K}, x / \varepsilon\right)\left(\nabla v_{M}-\nabla v^{H}\right)^{T} d x=0 .
\end{aligned}
$$

Equality (3.2) implies that $B\left(v^{H}, v^{H}\right) \geq C\left\|v^{H}\right\|_{H^{1}(K)}^{2}$, and thus the bilinear form $B$ is coercive. Equality (3.3) implies that $\left\|\nabla v_{M}\right\|_{L^{2}\left(K_{\varepsilon}\right)} \leq C\left\|\nabla v^{H}\right\|_{L^{2}\left(K_{\varepsilon}\right)}$, and it follows 
that $B$ is bounded. The existence and uniqueness of a solution $u^{H}$ of problem $(2.21)$ as well as (3.1) follow from the Lax-Milgram theorem.

The following representation for the solution $w_{M}$ of problem (2.20) will be useful in what follows.

LEMmA 3.2. Let $w_{M} \in S_{M}\left(K_{\varepsilon}\right)$ be the solution of problem (2.20). Then

$$
w_{M}=\varepsilon \sum_{j=1}^{d} \chi_{M}^{j}\left(x_{K}, x / \varepsilon\right) \frac{\partial u^{H}}{\partial x_{j}}
$$

where $\chi_{M}^{j}\left(x_{K}, y\right) \in S_{M}(Y), j=1, \ldots, d$, are the solutions of the problem

$$
\left(a\left(x_{K}, y\right) \nabla \chi_{M}^{j}, \nabla z_{M}\right)_{M}=\left(a\left(x_{K}, y\right) e_{j}, \nabla z_{M}\right)_{M} \quad \forall z_{M} \in S_{M}(Y),
$$

where $\left\{e_{j}\right\}_{j=1}^{d}$ is the standard basis of $\mathbb{R}^{d}$ and where we set $y=x / \varepsilon$.

Proof. We know from Proposition 3.1 that problems (2.20) and (3.5) have a unique solution. The lemma follows simply by inserting (3.4) into (2.20).

3.2. Convergence results. In this section, we estimate the convergence rate for the FES-HMM defined in section 2. In order to obtain convergence results for $\left\|u^{0}-u^{H}\right\|$, where $u^{0}$ is the solution of the homogenized problem (2.3) and $u^{H}$ is the solution of the FES-HMM defined in (2.21), we have to introduce the following semidiscrete bilinear form:

$$
\widetilde{B}\left(u^{H}, v^{H}\right)=\sum_{K \in \mathcal{T}_{H}} \frac{|K|}{\left|K_{\varepsilon}\right|} \int_{K_{\varepsilon}} \nabla u a\left(x_{K}, x / \varepsilon\right)(\nabla v)^{T} d x
$$

where $u$ (respectively, $v$ ) is the solution of the microproblem (2.22) such that $(u-$ $\left.u^{H}\right)=w \in W_{\text {per }}^{1}\left(K_{\varepsilon}\right)$. It can be shown similarly as in Lemma 3.2 that (see also [3] for details)

$$
w=\varepsilon \sum_{j=1}^{d} \chi^{j}\left(x_{K}, x / \varepsilon\right) \frac{\partial u^{H}}{\partial x_{j}}
$$

where $\chi^{j}\left(x_{K}, y\right) \in W_{p e r}^{1}(Y), j=1, \ldots, d$, are the solutions of the problem

$$
\int_{Y} \nabla \chi^{j} a\left(x_{K}, y\right)(\nabla z)^{T} d x=\int_{K_{\varepsilon}} e_{j}^{T} a\left(x_{K}, y\right)(\nabla z)^{T} d x \quad \forall z \in W_{p e r}^{1}(Y),
$$

where $\left\{e_{j}\right\}_{j=1}^{d}$ is the standard basis of $\mathbb{R}^{d}$ and where we set as usual $y=x / \varepsilon$. We define a semidiscrete macrosolution by $\widetilde{u}^{H}$, the solution of the following problem: find $\widetilde{u}^{H} \in S_{0}^{1}\left(\Omega, \mathcal{T}_{H}\right)$ such that

$$
\widetilde{B}\left(\widetilde{u}^{H}, v^{H}\right)=\left\langle f, v^{H}\right\rangle \quad \forall v^{H} \in S_{0}^{1}\left(\Omega, \mathcal{T}_{H}\right) .
$$

Similarly as for problem (2.21), it can be shown that this problem has a unique solution which satisfies $\left\|\widetilde{u}^{H}\right\|_{H^{1}(\Omega)} \leq C\|f\|_{L^{2}(\Omega)}$.

Following [3], the error estimates for the FES-HMM can be obtain as sketched below. Let $u^{H}, \widetilde{u}^{H}$ be the solution of problems (2.21) and (3.9), respectively. Let $u^{0}$ be the solution of the homogenized problem (2.3). Then

$$
\left\|u^{0}-u^{H}\right\|_{H^{1}(\Omega)} \leq C H\|f\|_{L^{2}(\Omega)}+\left\|\widetilde{u}^{H}-u^{H}\right\|_{H^{1}(\Omega)},
$$


where we used the triangle inequality and the estimation $\left\|u^{0}-\widetilde{u}^{H}\right\|_{H^{1}(\Omega)} \leq C H\|f\|_{L^{2}(\Omega)}$ (see [3]). The second term of the right-hand side of the inequality can be estimated in the following way. Denoting by $v^{H}=\widetilde{u}^{H}-u^{H}$, we have

$$
\alpha\left\|\widetilde{u}^{H}-u^{H}\right\|_{H^{1}(\Omega)}^{2} \leq \widetilde{B}\left(\widetilde{u}^{H}-u^{H}, v^{H}\right)=B\left(u^{H}, v^{H}\right)-\widetilde{B}\left(u^{H}, v^{H}\right)
$$

and thus

$$
\left\|\widetilde{u}^{H}-u^{H}\right\|_{H^{1}(\Omega)} \leq \frac{1}{\alpha} \frac{\left|B\left(u^{H}, v^{H}\right)-\widetilde{B}\left(u^{H}, v^{H}\right)\right|}{\left\|v^{H}\right\|_{H^{1}(\Omega)}} .
$$

This is the basis of our convergence analysis. For sufficiently smooth tensor $a(x, x / \varepsilon)$, we prove in what follows that the right-hand side of (3.12) is spectrally decaying, i.e., decays faster than any fixed degree polynomial rate. Provided analycity of $a^{\varepsilon}(x)$, we prove that the right-hand side of (3.12) is exponentially decaying. $L^{2}$ convergence rates will be derived as well.

3.3. Spectral and exponential convergence. We start with a lemma needed to derive the spectral accuracy of the method (see Appendix A for a proof).

LEMMA 3.3. Assume $w(y) \in H_{\text {per }}^{s}(Y)$, with $s \geq 2$; then

$$
\begin{aligned}
& \left\|w(y)-\mathbf{Q}_{M} w(y)\right\|_{L^{2}(Y)} \leq C M^{-s}\|w\|_{H^{s}(Y)}, \\
& \left\|\nabla w(y)-\nabla \mathbf{Q}_{M}(w(y))\right\|_{L^{2}(Y)} \leq C M^{1-s}\|w\|_{H^{s}(Y)},
\end{aligned}
$$

where for a function $w \in L_{\text {per }}^{2}(Y)$, the spectral interpolant $\mathbf{Q}_{M} w$ is defined in (2.11) and $M^{d}$ is the number of pseudospectral points of the microspace $S_{M}(Y)$ (see (2.15)).

For the proof of the exponential decay estimate, we first need some notation.

Definition 3.4. Let $I=(0,1)$. The set $\mathcal{A}_{B, \alpha}(I)$ consists of functions $g(y) \in$ $C^{\infty}(\mathbb{R}) I$-periodic and having an analytic extension in the strip

$$
\mathcal{E}_{\alpha}:=\{z \in \mathbb{C} ;|\operatorname{Im}(z)| \leq \alpha\} .
$$

We define $B:=\max _{z \in \mathcal{E}_{\alpha}}|g(z)|$. For a multivariate function $g(y) \in C^{\infty}(\mathbb{R}) Y$ periodic, where $Y=(0,1)^{d}$, we say that $g(y)$ belongs to $\mathcal{A}_{\mathcal{B}, \alpha}(Y)$ if for all $i=1, \ldots, d$ and each $Y_{-i}=\left(y_{1}, \ldots, y_{i-1}, y_{i+1}, \ldots, y_{d}\right) \in(0,1)^{d-1}, g_{i}\left(y_{i}\right):=g\left(Y_{-i}, y_{i}\right)$ has an analytic extension in the strip $\mathcal{E}_{\alpha}$. We denote by $\mathcal{B}:=\max _{i \leq d}\left\{\max _{Y_{-i}}\left\{\max _{z \in \mathcal{E}_{\alpha}}\left|g\left(Y_{-i}, z\right)\right|\right\}\right\}$.

REMARK 3.5. It is known that if the coefficients of an elliptic operator are real analytic in the closure of the domain of consideration $\bar{D}$ and if the right-hand side is real analytic, then the solution of the corresponding elliptic equation is a real analytic function in $\bar{D}$. We refer the reader to [27], [9] for a precise discussion and proof of the above statement. For the problem (3.8), we note that the coefficients of the elliptic operator and the right-hand side are given by the same functions $a_{i j}\left(x_{K}, \cdot\right)$. Assuming $a_{i j}\left(x_{K}, \cdot\right) \in \mathcal{A}_{\mathcal{B}, \alpha}(Y)$ for all $i, j=1, \ldots, d$, it follows from the above consideration that the solutions $\chi^{j}\left(x_{K}, y\right)$ of $(3.8)$ are also analytic for all $j=1, \ldots, d$.

The following lemma is proved in Appendix A.

Lemma 3.6. Let $w \in \mathcal{A}_{\mathcal{B}, \alpha}(Y)$. Then

$$
\begin{aligned}
& \left\|w(y)-\mathbf{Q}_{M} w(y)\right\|_{L^{2}(Y)} \leq C \mathcal{B}(\log M)^{d-1} e^{-\alpha M}, \\
& \left\|\nabla w(y)-\nabla \mathbf{Q}_{M}(w(y))\right\|_{L^{2}(Y)} \leq C \mathcal{B} M(\log M)^{d-1} e^{-\alpha M},
\end{aligned}
$$

where for a function $w \in L_{p e r}^{2}(Y)$, the spectral interpolant $\mathbf{Q}_{M} w$ is defined in (2.11) and $M^{d}$ is the number of pseudospectral points of the microspace $S_{M}(Y)$ (see (2.15)).

Copyright $@$ by SIAM. Unauthorized reproduction of this article is prohibited. 
Let $a^{\varepsilon}=\left(a_{i j}\left(x_{K}, x / \varepsilon\right)\right)_{i, j=1}^{d}$ be the bounded coercive tensor of problem (2.1). We set $a_{i j}\left(x_{K}, x / \varepsilon\right)=a_{i j}\left(x_{K}, y\right)$. In view of (3.10) and (3.12), we prove the main results of this section, leading to spectral and exponential convergence of the microsolver.

Lemma 3.7. Assume that $a_{i j}(x, y), X^{j}(x, y) \in L^{\infty}\left(\Omega ; H_{p e r}^{s}(Y)\right)$ for all $i, j=$ $1, \ldots, d$ with $s>2$. Then, for $u^{H}, v^{H} \in S_{0}^{1}\left(\Omega, \mathcal{T}_{H}\right)$,

$$
\left|B\left(u^{H}, v^{H}\right)-\widetilde{B}\left(u^{H}, v^{H}\right)\right| \leq C\left(M^{1-s}\right)^{2}\left\|\nabla u^{H}\right\|_{L^{2}(\Omega)}\left\|\nabla v^{H}\right\|_{L^{2}(\Omega)},
$$

where $C$ depends on $\left\|a_{i j}\right\|_{L^{\infty}\left(\Omega ; H_{p e r}^{s}(Y)\right)},\left\|\chi^{j}\right\|_{L^{\infty}\left(\Omega ; H_{p e r}^{s}(Y)\right)}$, where the bilinear forms $B(\cdot, \cdot), \widetilde{B}(\cdot, \cdot)$ are defined in (2.19) and (3.6), respectively, and where $M^{d}$ is the number of pseudospectral points of the microspace $S_{M}\left(K_{\varepsilon}\right)$ given in (2.15).

LEMmA 3.8. Assume that $\chi^{j}(x, \cdot), a_{i, j}(x, \cdot) \in \mathcal{A}_{\mathcal{B}, \alpha}(Y)$ for all $i, j=1, \ldots, d$ and a.e. $x \in \Omega$. Then, for $u^{H}, v^{H} \in S_{0}^{1}\left(\Omega, \mathcal{T}_{H}\right)$,

$$
\left|B\left(u^{H}, v^{H}\right)-\widetilde{B}\left(u^{H}, v^{H}\right)\right| \leq C\left(M(\log M)^{(d-1)} e^{-\alpha M}\right)^{2}\left\|\nabla u^{H}\right\|_{L^{2}(\Omega)}\left\|\nabla v^{H}\right\|_{L^{2}(\Omega)},
$$

where the constant $C$ depends on $\mathcal{B}$, where the bilinear forms $B(\cdot, \cdot), \widetilde{B}(\cdot, \cdot)$ are defined in (2.19) and (3.6), respectively, and where $M^{d}$ is the number of pseudospectral points of the microspace $S_{M}\left(K_{\varepsilon}\right)$ given in (2.15).

The proof of both lemmas follows the same lines. We prove Lemma 3.8 and indicate after the proof the modifications which lead to the proof of Lemma 3.7.

Proof of Lemma 3.8. Using the definitions of (2.19) and (3.6), following Lemma 3.3 of [3] we have

$$
\begin{aligned}
& \left|B\left(u^{H}, v^{H}\right)-\widetilde{B}\left(u^{H}, v^{H}\right)\right| \\
= & \left|\sum_{K \in \mathcal{T}} \frac{|K|}{\left|K_{\varepsilon}\right|}\left(\int_{K_{\varepsilon}} \nabla u a\left(x_{K}, x / \varepsilon\right)(\nabla v)^{T} d x-\int_{K_{\varepsilon}} \nabla u_{M} a\left(x_{K}, x / \varepsilon\right)\left(\nabla v_{M}\right)^{T} d x\right)\right| \\
= & \mid \sum_{K \in \mathcal{T}} \frac{|K|}{\left|K_{\varepsilon}\right|}\left(\int_{K_{\varepsilon}} \nabla\left(u-u_{M}\right) a\left(x_{K}, x / \varepsilon\right)(\nabla v)^{T} d x\right. \\
& \left.-\int_{K_{\varepsilon}} \nabla u_{M} a\left(x_{K}, x / \varepsilon\right)\left(\nabla\left(v_{M}-v\right)\right)^{T} d x\right) \mid .
\end{aligned}
$$

We observe that the first member of the last line of (3.20) is zero since $\left(u-v_{M}\right) \in$ $W_{p e r}^{1}\left(K_{\varepsilon}\right)$. Using the same argument and replacing $u_{M}$ by $u_{M}-u$ in the second expression, we have

$$
\begin{aligned}
& \left|\sum_{K \in \mathcal{T}} \frac{|K|}{\left|K_{\varepsilon}\right|} \int_{K_{\varepsilon}} \nabla\left(u_{M}-u\right) a\left(x_{K}, x / \varepsilon\right)\left(\nabla\left(v_{M}-v\right)\right)^{T} d x\right| \\
& \leq C \sum_{K \in \mathcal{T}} \frac{|K|}{\left|K_{\varepsilon}\right|}\left\|\nabla u_{M}-\nabla u\right\|_{L^{2}\left(K^{\varepsilon}\right)}\left\|\nabla v_{M}-\nabla v\right\|_{L^{2}\left(K^{\varepsilon}\right)},
\end{aligned}
$$

Copyright (c) by SIAM. Unauthorized reproduction of this article is prohibited. 
where we used that the bilinear form is bounded. We have next to estimate

$$
\begin{aligned}
& \left\|\nabla u_{M}-\nabla u\right\|_{L^{2}\left(K_{\varepsilon}\right)}=\left\|\varepsilon \sum_{j=1}^{d} \nabla\left(\chi_{M}^{j}\left(x_{K}, x / \varepsilon\right)-\chi^{j}\left(x_{K}, x / \varepsilon\right)\right) \frac{\partial u^{H}}{\partial x_{j}}\right\|_{L^{2}\left(K_{\varepsilon}\right)} \\
& =\sqrt{\left|K_{\varepsilon}\right|}\left\|\sum_{j=1}^{d} \nabla\left(\chi_{M}^{j}\left(x_{K}, y\right)-\chi^{j}\left(x_{K}, y\right)\right) \frac{\partial u^{H}}{\partial x_{j}}\right\| \|_{L^{2}(Y)} \\
& \leq C \sqrt{\left|K_{\varepsilon}\right|}\left\|\nabla u^{H}\right\|_{L^{2}(Y)} \max _{j}\left\|\nabla\left(\chi_{M}^{j}-\chi^{j}\right)\right\|_{L^{2}(Y)} .
\end{aligned}
$$

It remains to estimate $\left\|\nabla\left(\chi_{M}^{j_{m}}-\chi^{j_{m}}\right)\right\|_{L^{2}(Y)}$, where $j_{m}$ is the index corresponding to the maximum in the above expression. In view of (3.5) and (3.8), using the Strang lemma we get

$$
\begin{aligned}
& \left\|\nabla\left(\chi_{M}^{j_{m}}-\chi^{j_{m}}\right)\right\|_{L^{2}(Y)} \leq C\left(\operatorname { i n f } _ { z _ { M } \in S _ { M } ( Y ) } \left(\left\|\nabla\left(\chi^{j_{m}}-z_{M}\right)\right\|_{L^{2}(Y)}\right.\right. \\
& \left.+\sup _{w_{M} \in S_{M}(Y)} \frac{\left|\left(a\left(x_{K}, y\right)\left(\nabla z_{M}\right)^{T}, \nabla w_{M}\right)-\left(a\left(x_{K}, y\right)\left(\nabla z_{M}\right)^{T}, \nabla w_{M}\right)_{M}\right|}{\left\|\nabla w_{M}\right\|_{L^{2}(Y)}}\right) \\
& \left.+\sup _{w_{M} \in S_{M}(Y)} \frac{\left|\left(a\left(x_{K}, y\right) e_{j_{m}}, \nabla w_{M}\right)-\left(a\left(x_{K}, y\right) e_{j_{m}}, \nabla w_{M}\right)_{M}\right|}{\left\|\nabla w_{M}\right\|_{L^{2}(Y)}}\right) .
\end{aligned}
$$

We chose $z_{M}=\chi_{M}^{j_{m}}$ for the infimum in (3.24), and using Lemma 3.6 we obtain

$$
\left\|\nabla\left(\chi^{j_{m}}-\chi_{M}^{j_{m}}\right)\right\|_{L^{2}(Y)} \leq C \mathcal{B} M(\log M)^{d-1} e^{-\alpha M} .
$$

For the second term of the right-hand side of (3.24) (with $z_{M}=\chi_{M}^{j_{m}}$ ), we have

$$
\begin{aligned}
& \left|\left(a\left(x_{K}, y\right)\left(\nabla \chi_{M}^{j_{m}}\right)^{T}, \nabla w_{M}\right)-\left(a\left(x_{K}, y\right)\left(\nabla \chi_{M}^{j_{m}}\right)^{T}, \nabla w_{M}\right)_{M}\right| \\
& \quad \leq\left|\left(\left(a\left(x_{K}, y\right)-\mathbf{Q}_{M}\left(a\left(x_{K}, y\right)\right)\right)\left(\nabla \chi_{M}^{j_{m}}\right)^{T}, \nabla w_{M}\right)\right| \\
& \quad+\left|\left(\left(a\left(x_{K}, y\right)-\mathbf{Q}_{M}\left(a\left(x_{K}, y\right)\right)\right)\left(\nabla \chi_{M}^{j_{m}}\right)^{T}, \nabla w_{M}\right)_{M}\right|
\end{aligned}
$$

where we used Remark 2.2. The second term is zero, and the first term is bounded by

$$
\begin{aligned}
& \left|\left(\left(a\left(x_{K}, y\right)-\mathbf{Q}_{M}\left(a\left(x_{K}, y\right)\right)\right)\left(\nabla \chi_{M}^{j_{m}}\right)^{T}, \nabla w_{M}\right)\right| \\
& \leq\left\|\nabla \chi_{M}^{j_{m}}\right\|_{L^{\infty}(Y)} \int_{Y} \sum_{l, m=1}^{d}\left|\left(a_{l, m}\left(x_{K}, y\right)-\mathbf{Q}_{M}\left(a_{l, m}\left(x_{K}, y\right)\right)\right) \partial_{y_{l}} w_{M}\right| d y \\
& \leq\left\|\nabla \chi_{M}^{j_{m}}\right\|_{L^{\infty}(Y)} \max _{l, m}\left\|a_{l, m}\left(x_{K}, y\right)-\mathbf{Q}_{M}\left(a_{l, m}\left(x_{K}, y\right)\right)\right\|_{L^{2}(Y)}\left\|\nabla w_{M}\right\|_{L^{2}(Y)} \\
& \leq C \mathcal{B}(\log M)^{d-1} e^{-\alpha M}\left\|\nabla w_{M}\right\|_{L^{2}(Y)}
\end{aligned}
$$

using the assumptions and Lemma 3.6. For the third term of the right-hand side of (3.24), we write $g_{j_{m}}=a\left(x_{K}, y\right) e_{j_{m}}$ and $\mathbf{Q}_{M} g_{j_{m}}$, the spectral interpolant of $g_{j_{m}}$ in $S_{M}(Y)$. Using Remark 2.2 and Lemma 3.6 we obtain

$$
\begin{aligned}
& \left|\left(g_{j_{m}}, \nabla w_{M}\right)-\left(g_{j_{m}}, \nabla w_{M}\right)_{M}\right|=\left|\left(g_{j_{m}}-\mathbf{Q}_{M}\left(g_{j_{m}}\right), \nabla w_{M}\right)\right| \\
& \leq\left\|g_{j_{m}}-\mathbf{Q}_{M}\left(g_{j_{m}}\right)\right\|_{L^{2}(Y)}\left\|\nabla w_{M}\right\|_{L^{2}(Y)} \leq C \mathcal{B} e^{-\alpha M}\left\|\nabla w_{M}\right\|_{L^{2}(Y)}
\end{aligned}
$$

Copyright $@$ ㅇ by SIAM. Unauthorized reproduction of this article is prohibited. 
using Lemma 3.6. Summing all the terms together and using that $\nabla u^{H}$ is constant, we obtain

$$
\begin{aligned}
& \sqrt{|K| /\left|K_{\varepsilon}\right|}\left\|\nabla u_{M}-\nabla u\right\|_{L^{2}\left(K_{\varepsilon}\right)} \leq C \mathcal{B} M(\log M)^{d-1} e^{-\alpha M} \sqrt{|K|}\left\|\nabla u^{H}\right\|_{L^{2}(Y)} \\
& \leq C \mathcal{B} M(\log M)^{d-1} e^{-\alpha M}\left\|\nabla u^{H}\right\|_{L^{2}(K)} .
\end{aligned}
$$

The second term of (3.22), $\left\|\nabla v_{M}-\nabla v\right\|_{L^{2}\left(K_{\varepsilon}\right)}$, is treated similarly. Finally, summing up over $K \in \mathcal{T}$ we find that (3.22) is bounded by

$$
C\left(\mathcal{B} M(\log M)^{d-1} e^{-\alpha M}\right)^{2}\left\|\nabla v^{H}\right\|_{L^{2}(\Omega)}\left\|\nabla w^{H}\right\|_{L^{2}(\Omega)},
$$

and the proof is complete.

Proof of Lemma 3.7. The first part of the proof until inequality (3.24) is identical as for Lemma 3.8. We next discuss the estimation of the three terms on the right-hand side of the inequality (3.24). We chose $z_{M}=\chi_{M}^{j_{m}}$ for the infimum in (3.24), and the first term can be bounded using Lemma 3.3 by

$$
\left\|\nabla\left(\chi^{j_{m}}-\chi_{M}^{j_{m}}\right)\right\|_{L^{2}(Y)} \leq C M^{1-s}\left\|\chi^{j_{m}}\left(x_{K}, y\right)\right\|_{H^{s}(Y)} .
$$

For the second term on the right-hand side of (3.24) (with $z_{M}=\chi_{M}^{j_{m}}$ ), Lemma 3.3 leads to

$$
\begin{aligned}
& \left|\left(a\left(x_{K}, y\right)\left(\nabla \chi_{M}^{j_{m}}\right)^{T}, \nabla w_{M}\right)-\left(a\left(x_{K}, y\right)\left(\nabla \chi_{M}^{j_{m}}\right)^{T}, \nabla w_{M}\right)_{M}\right| \\
& \leq C M^{1-s} \max _{l, m}\left\|a_{l, m}\left(x_{K}, y\right)\right\|_{H^{s}(Y)}\left\|\nabla \chi^{j_{m}}\left(x_{K}, y\right)\right\|_{L^{\infty}(Y)}\left\|\nabla w_{M}\right\|_{L^{2}(Y)},
\end{aligned}
$$

which is obtained similarly as in Lemma 3.8. Notice that since $\chi^{j}(x, y) \in L^{\infty}\left(\Omega ; H_{p e r}^{s}(Y)\right)$ with $s>2$, we have that $\chi^{j}(x, y) \in L^{\infty}\left(\Omega ; W^{1, \infty}(Y)\right)$ (Sobolev's inequality). For the third term of the right-hand side of (3.24), we write $g_{j_{m}}=a\left(x_{K}, y\right) e_{j_{m}}$ and $\mathbf{Q}_{M} g_{j_{m}}$, the interpolant of $g j_{m}$ in $S_{M}(Y)$. Similarly as in Lemma 3.8 and using Lemma 3.3, we obtain

$$
\begin{aligned}
& \left|\left(g_{j_{m}}, \nabla w_{M}\right)-\left(g_{j_{m}}, \nabla w_{M}\right)_{M}\right|=\left|\left(g_{j_{m}}-\mathbf{Q}_{M}\left(g_{j_{m}}\right), \nabla w_{M}\right)\right| \\
& \leq C M^{-s} \max _{l, m}\left\|a_{l, m}\left(x_{K}, y\right)\right\|_{H^{s}(Y)}\left\|\nabla w_{M}\right\|_{L^{2}(Y)} .
\end{aligned}
$$

Summing all the terms together and using that $\nabla u^{H}$ is constant, we obtain

$$
\sqrt{|K| /\left|K_{\varepsilon}\right|}|| \nabla u_{M}-\nabla u\left\|_{L^{2}\left(K_{\varepsilon}\right)} \leq M^{1-s}\right\| \nabla u^{H} \|_{L^{2}(Y)} .
$$

Using similar arguments for the second term of (3.22), summing over $K \in \mathcal{T}$ and using that $a_{i j}(x, y), \chi^{j}(x, y) \in L^{\infty}\left(\Omega ; H_{p e r}^{s}(Y)\right)$ we find that $(3.22)$ is bounded by

$$
C\left(M^{1-s}\right)^{2}\left\|\nabla u^{H}\right\|_{L^{2}(\Omega)}\left\|\nabla v^{H}\right\|_{L^{2}(\Omega)},
$$

and the proof is complete.

3.4. Error estimates for the FES-HMM. We can now give the error estimate between the homogenized solution and the solution of the FES-HMM.

ThEOREM 3.9. Let $u^{0}$ be the solution of the homogenized problem (2.3), and assume $u^{0}$ is $H^{2}$-regular. Let $u^{H}$ be the solution of problem (2.21), and suppose that the assumptions of Lemma 3.3 hold. Then

$$
\left\|u^{0}-u^{H}\right\|_{H^{1}(\Omega)} \leq\left(C_{1} H+C_{2} M^{2(1-s)}\right)\|f\|_{L^{2}(\Omega)},
$$

Copyright $@$ ㅇ by SIAM. Unauthorized reproduction of this article is prohibited. 
where $C_{2}$ depends on $\left\|a_{i j}\right\|_{L^{\infty}\left(\Omega ; H_{p e r}^{s}(Y)\right)},\left\|\chi^{j}\right\|_{L^{\infty}\left(\Omega ; H_{\text {per }}^{s}(Y)\right)}$, where $H$ is the size of the triangulation of the macro FE space (2.13), and where $M^{d}$ is the number of pseudospectral points of the microspace $S_{M}\left(K_{\varepsilon}\right)$ given in (2.15).

Proof. Using (3.10), (3.12), and Lemma 3.3 gives the result.

Corollary 3.10. Suppose that the assumptions of Theorem 3.9 hold. Then we have the following estimates:

$$
\begin{aligned}
& \left\|u^{0}-u^{H}\right\|_{L^{2}(\Omega)} \leq\left(C_{1} H^{2}+C_{2} M^{2(1-s)}\right)\|f\|_{L^{2}(\Omega)}, \\
& \left\|u^{\varepsilon}-u^{H}\right\|_{L^{2}(\Omega)} \leq\left(C_{1} H^{2}+C_{2} M^{2(1-s)}+C_{3} \varepsilon\right)\|f\|_{L^{2}(\Omega)} .
\end{aligned}
$$

Proof. Estimation (3.26) follows from the estimation $\left\|u^{0}-\widetilde{u}^{H}\right\|_{L^{2}(\Omega)} \leq C H^{2}\|f\|_{L^{2}(\Omega)}$ for the solution of (3.9) (see [2, Appendix A]), the estimation for (3.12) obtained in Lemma 3.3, and the triangle inequality. Estimation (3.27) follows from (2.5), (3.26), and the triangle inequality.

Using Lemma 3.6 instead of Lemma 3.3 we obtain the following theorem.

THEOREM 3.11. Let $u^{0}$ be the solution of the homogenized problem (2.3), and assume $u^{0}$ is $H^{2}$-regular. Let $u^{H}$ be the solution of problem (2.21), and suppose that the assumptions of Lemma 3.6 hold. Then

$$
\left\|u^{0}-u^{H}\right\|_{H^{1}(\Omega)} \leq\left(C_{1} H+C_{2}\left(M(\log M)^{(d-1)} e^{-\alpha M}\right)^{2}\right)\|f\|_{L^{2}(\Omega)},
$$

where $C_{2}$ depends on $\mathcal{B}$, where $H$ is the size of the triangulation of the macro $F E$ space (2.13), and where $M^{d}$ is the number of pseudospectral points of the microspace $S_{M}\left(K_{\varepsilon}\right)$ given in $(2.15)$.

COROLlary 3.12. Suppose that the assumptions of Theorem 3.11 hold. Then we have the following estimates:

$$
\begin{aligned}
& \left\|u^{0}-u^{H}\right\|_{L^{2}(\Omega)} \leq\left(C_{1} H^{2}+C_{2}\left(M(\log M)^{(d-1)} e^{-\alpha M}\right)^{2}\right)\|f\|_{L^{2}(\Omega)}, \\
& \left\|u^{\varepsilon}-u^{H}\right\|_{L^{2}(\Omega)} \leq\left(C_{1} H^{2}+C_{2}\left(M(\log M)^{(d-1)} e^{-\alpha M}\right)^{2}+C_{3} \varepsilon\right)\|f\|_{L^{2}(\Omega)} .
\end{aligned}
$$

3.5. Retrieving microscopic information. So far we gave a numerical procedure to approximate the macro- (homogenized) solution of the problem (2.3). Following [28] (see also [14], [3]) we consider a procedure to retrieve the microscopic information in order to approximate the microscopic solution of problem (2.1). We define $u_{p}^{\varepsilon}$ by

$$
\left.u_{p}^{\varepsilon}(x)\right|_{K}=u^{H}(x)+\left.\left(u_{M}(x)-u^{H}(x)\right)\right|_{K} ^{P} \quad \text { for } x \in K \in \mathcal{T}_{H},
$$

where $\left.\right|_{K} ^{P}$ denotes the periodic extension of the fine scale solution $\left(u_{M}-u^{H}\right)$, available in $K_{\varepsilon}$, on each element $K$. This extension is defined for a function $w \in H^{1}\left(K_{\varepsilon}\right)$ or in $\bar{S}_{M}\left(K_{\varepsilon}\right)$ by

$$
w_{p}(x+\varepsilon j)=w(x) \quad \forall j=\left(j_{l}, \ldots, j_{d}\right) \in \mathbb{Z}^{d}, \forall x \in K_{\varepsilon} \quad \text { such that } x+\varepsilon j \in K .
$$

The function $u_{p}^{\varepsilon}$, which can be expressed by

$$
u_{p}^{\varepsilon}=u^{H}(x)+\left.\varepsilon \sum_{j=1}^{d} \chi_{M}^{j}\left(x_{K}, x / \varepsilon\right)\right|_{K} ^{P} \frac{\partial u^{H}}{\partial x_{j}}
$$

Copyright $@$ by SIAM. Unauthorized reproduction of this article is prohibited. 
using (3.4) for the whole macroelement $K$, defines a fully discrete fine scale approximation of the solution $u^{\varepsilon}$ of problem (2.1). Since $u_{p}^{\varepsilon}$ can be discontinuous across the macroelements $K$, we define a broken $H^{1}$ norm by

$$
\|u\|_{\bar{H}^{1}(\Omega)}:=\left(\sum_{K \in \mathcal{T}_{H}}\|\nabla u\|_{L^{2}(K)}^{2}\right)^{1 / 2} .
$$

Similarly as in (3.32), we define

$$
\widetilde{u}_{p}^{\varepsilon}=\widetilde{u}^{H}(x)+\left.\varepsilon \sum_{j=1}^{d} \chi^{j}\left(x_{K}, x / \varepsilon\right)\right|_{K} ^{P} \frac{\partial \widetilde{u}^{H}}{\partial x_{j}},
$$

the reconstructed semidiscrete function based on the formula (3.7), where $\widetilde{u}^{H}$ is the solution of problem (3.9). In what follows we will skip the notation $\left.\right|_{K} ^{P}$ for the functions defined in (3.32) and (3.34). We obtain an error estimate of the fully discrete fine scale solution (3.31) in the following way:

$$
\left\|u^{\varepsilon}-u_{p}^{\varepsilon}\right\|_{\bar{H}^{1}(\Omega)} \leq\left\|u^{\varepsilon}-\widetilde{u}_{p}^{\varepsilon}\right\|_{\bar{H}^{1}(\Omega)}+\left\|\widetilde{u}_{p}^{\varepsilon}-u_{p}^{\varepsilon}\right\|_{\bar{H}^{1}(\Omega)} .
$$

The first term of the right-hand side of the above inequality can be bounded by $\left\|u^{\varepsilon}-\widetilde{u}_{p}^{\varepsilon}\right\|_{\bar{H}^{1}(\Omega)} \leq C(\sqrt{\varepsilon}+H)\|f\|_{L^{2}(\Omega)}$ (see [14], [3]), and it remains to estimate the second term. We have

$$
\begin{aligned}
& \sum_{K \in \mathcal{T}_{H}}\left\|\nabla\left(\widetilde{u}_{p}^{\varepsilon}-u_{p}^{\varepsilon}\right)\right\|_{L^{2}(K)}^{2} \leq \sum_{K \in \mathcal{T}_{H}}\left\|\nabla\left(\widetilde{u}^{H}-u^{H}\right)\right\|_{L^{2}(K)}^{2} \\
& +\sum_{K \in \mathcal{T}_{H}}\left\|\sum_{j=1}^{n} \nabla\left(\varepsilon \chi^{j}\left(x_{K}, x / \varepsilon\right)\right)\left(\frac{\partial \widetilde{u}^{H}}{\partial x_{j}}-\frac{\partial u^{H}}{\partial x_{j}}\right)\right\|_{L^{2}(K)}^{2} \\
& +\sum_{K \in \mathcal{T}_{H}}\left\|\sum_{j=1}^{n} \nabla\left(\varepsilon\left(\chi^{j}-\chi_{M}^{j}\right)\left(x_{K}, x / \varepsilon\right)\right) \frac{\partial u^{H}}{\partial x_{j}}\right\|_{L^{2}(K)}^{2}
\end{aligned}
$$

The first two terms are bounded by $C M^{2}(\log M)^{2(d-1)} e^{-2 \alpha M}\|f\|_{L^{2}(\Omega)}^{2}$ if the assumptions of Lemma 3.8 hold and by $C M^{2(1-s)}\|f\|_{L^{2}(\Omega)}$ if the assumptions of Lemma 3.7 hold. For the last term, we take a finite covering of $K \subset \bigcup_{x_{l} \in K} K_{\varepsilon}\left(x_{l}\right)$, where $K_{\varepsilon}\left(x_{l}\right)=x_{l}+\varepsilon[-1 / 2,1 / 2]^{d}$, and bound it by $C M^{2}(\log M)^{2(d-1)} e^{-2 \alpha M}\left\|\nabla u^{H}\right\|_{L^{2}(\Omega)}^{2}$ using Lemma 3.6 or by $C M^{2(1-s)}\left\|\nabla u^{H}\right\|_{L^{2}(\Omega)}^{2}$ with Lemma 3.3 .

Using the norm defined in (3.33) and (3.1), we obtain

$\left\|\widetilde{u}_{p}^{\varepsilon}-u_{p}^{\varepsilon}\right\|_{\bar{H}^{1}(\Omega)} \leq C M(\log M)^{d-1} e^{-\alpha M}\|f\|_{L^{2}(\Omega)}$ or $\left\|u_{p}^{\varepsilon}-u_{p}^{\varepsilon}\right\|_{\bar{H}^{1}(\Omega)} \leq C M^{1-s}\|f\|_{L^{2}(\Omega)}$

if the assumptions of Lemma 3.6 or Lemma 3.3 hold, respectively. We have proved the following theorem.

TheOREM 3.13. Let $u_{p}^{\varepsilon}$ be defined by (3.31) and $u^{\varepsilon}$ be the solution of (2.1). Suppose that the assumptions of Lemma 3.3 hold. Then

$$
\left\|u^{\varepsilon}-u_{p}^{\varepsilon}\right\|_{\bar{H}^{1}(\Omega)} \leq\left(C_{1} H+C_{2} M^{1-s}+C_{3} \sqrt{\varepsilon}\right)\|f\|_{L^{2}(\Omega)},
$$

where $C_{2}$ depends on $\left\|a_{i j}\right\|_{L^{\infty}\left(\Omega ; H_{p e r}^{s}(Y)\right)},\left\|\chi^{j}\right\|_{L^{\infty}\left(\Omega ; H_{p e r}^{s}(Y)\right)}$, where $H$ is the size of the triangulation of the macro FE space (2.13), and where $M^{d}$ is the number of pseudospectral points of the microspace $S_{M}\left(K_{\varepsilon}\right)$ given in (2.15).

Copyright (c) by SIAM. Unauthorized reproduction of this article is prohibited. 
TheOREM 3.14. Let $u_{p}^{\varepsilon}$ be defined by (3.31) and $u^{\varepsilon}$ be the solution of (2.1). Suppose that the assumptions of Lemma 3.6 hold. Then

$$
\left\|u^{\varepsilon}-u_{p}^{\varepsilon}\right\|_{\bar{H}^{1}(\Omega)} \leq\left(C_{1} H+C_{2} M(\log M)^{d-1} e^{-\alpha M}+C_{3} \sqrt{\varepsilon}\right)\|f\|_{L^{2}(\Omega)},
$$

where $C_{2}$ depends on $\mathcal{B}$, where $H$ is the size of the triangulation of the macro $F E$ space (2.13), and where $M^{d}$ is the number of pseudospectral points of the microspace $S_{M}\left(K_{\varepsilon}\right)$ given in (2.15).

4. Higher order macroscopic solver. In this section we discuss higher order multiscale methods. We consider a macro FE space defined by

$$
S_{0}^{p}\left(\Omega, \mathcal{T}_{H}\right)=\left\{u^{H} \in H_{0}^{1}(\Omega) ;\left.u^{H}\right|_{K} \in \mathcal{P}^{p}(K) \forall K \in \mathcal{T}_{H}\right\},
$$

where $\mathcal{P}^{p}(K)$ is the space of polynomials of degree $p \geq 1$ on the triangle $K$, and $\mathcal{T}_{H}$ is a quasi-uniform triangulation of $\Omega \subset \mathbb{R}^{d}$ of shape regular triangles $K$. We further assume that the solution of the homogenized problem (2.3) satisfies $u^{0} \in H^{l+1}(\Omega)$ and that the right-hand side satisfies $f \in H^{l}(\Omega)$.

We also consider a $p$ th-order $(p>1)$ numerical quadrature scheme $\left(\omega_{i}, x_{i}\right), i=$ $1, \ldots, L$, on $K$, with $\omega_{i}>0$ for all $i$ such that ${ }^{1}$

$$
\frac{1}{|K|} \int_{K} p(x) d x=\sum_{i=1}^{L} \omega_{i} p\left(x_{i}\right) \quad \forall p(x) \in \mathcal{P}^{2 p-2}(K) .
$$

For $u^{H} \in S_{0}^{p}\left(\Omega, \mathcal{T}_{H}\right)$ we define its linear approximation at a quadrature point $x_{i}$ by

$$
u_{\text {lin }}^{H}:=u^{H}\left(x_{i}\right)+\nabla u^{H}\left(x_{i}\right) \cdot\left(x-x_{i}\right) .
$$

Following [14], we define a bilinear form with the help of the quadrature formula by

$$
B\left(u^{H}, v^{H}\right)=\sum_{K \in \mathcal{T}_{H}} \sum_{i=1}^{L} \omega_{i} \int_{K_{\varepsilon}, i} \nabla u_{l i n, M} a\left(x_{i}, x / \varepsilon\right)\left(\nabla v_{l i n, M}\right)^{T} d x,
$$

where $K_{\varepsilon, i}=x_{i}+\varepsilon[-1 / 2,1 / 2]^{d} \subset K$ is a sampling subdomain centered at the quadrature point $x_{i}$ and $u_{l i n, M}$ is such that $\left(u_{l i n, M}-u_{l i n}^{H}\right)=w_{M} \in S_{M}\left(K_{\varepsilon}\right)$ and $w_{M}$ is the solution of the microproblem defined in (2.20).

The macrosolution of the FES-HMM is then given by the following variational problem: find $u^{H} \in S_{0}^{p}\left(\Omega, \mathcal{T}_{H}\right)$ such that

$$
B\left(u^{H}, v^{H}\right)=\left\langle f, v^{H}\right\rangle \quad \forall v^{H} \in S_{0}^{p}\left(\Omega, \mathcal{T}_{H}\right) .
$$

Following the line of Proposition 3.1 shows that the problem (4.5) is well-posed. Notice that the assumptions on the quadrature formula (4.2) are needed to ensure the coercivity of the bilinear form (4.4) (see [11, Theorem 4.1.2]).

As in section 3 , we consider the semidiscrete bilinear form $\widetilde{B}(\cdot, \cdot)$ similar to (4.4) but with microsolution $\left(u-u_{\text {lin }}^{H}\right)=w \in W_{p e r}^{1}\left(K_{\varepsilon}\right)$ and the semidiscrete solution $\widetilde{u}^{H} \in S_{0}^{l}\left(\Omega, \mathcal{T}_{H}\right)$ of the corresponding problem (3.9). Let $u^{0}$ be the solution of the homogenized problem (2.3). Then

$$
\left\|u^{0}-u^{H}\right\|_{H^{1}(\Omega)} \leq\left\|u^{0}-\widetilde{u}\right\|_{H^{1}(\Omega)}+\left\|\widetilde{u}-u^{H}\right\|_{H^{1}(\Omega)} .
$$

\footnotetext{
${ }^{1}$ We assume, of course, that the quadrature points are of type PI (positive interior), i.e., that $\omega_{i}>0, x_{i} \in K$ for all $i$.
}

Copyright $@$ by SIAM. Unauthorized reproduction of this article is prohibited. 
It has been shown in [14], provided $u^{0} \in H^{l+1}(\Omega)$, that $\left\|u^{0}-\widetilde{u}\right\|_{H^{1}(\Omega)} \leq C\left(H^{\min (p, l)}+\right.$ $\varepsilon)$ (see also [11, Theorem 4.1.6] for a discussion on the regularity assumptions).

In the above estimate and in what follows, $H^{l+1}(\Omega)$ regularity of the homogenized problem and $f \in H^{l-1}(\Omega)$ is assumed. If this does not hold, the above constant $C$ $\left(C_{1}\right.$ in what follows) should depend on $\left|u^{0}\right|_{H^{l+1}(\Omega)}$.

REMARK 4.1. The above result has been shown for a modified bilinear form similar to (4.4) but where the tensor $a(x, x / \varepsilon)$ is not collocated at the integration points. For the bilinear form (4.4), following [2, Appendix A] and the proof in [14] it can be shown that

$$
\left\|u^{0}-\widetilde{u}\right\|_{H^{1}(\Omega)} \leq C H^{\min (p, l)} .
$$

The remaining term $\left\|\widetilde{u}-u^{H}\right\|_{H^{1}(\Omega)}$ can be estimated following the proof of Lemmas 3.8 and 3.7. Furthermore, we can define a reconstructed solution similarly as in (3.31). We summarize our discussion.

THEOREM 4.2. Let $u^{0}$ be the solution of the homogenized problem (2.3), and assume $u^{0} \in H^{l+1}(\Omega)$. Let $u^{H}$ be the solution of problem (4.5), and suppose that the assumptions of Lemma 3.3 hold. Then

$$
\begin{aligned}
& \left\|u^{0}-u^{H}\right\|_{H^{1}(\Omega)} \leq C_{1} H^{\min (p, l)}+C_{2} M^{2(1-s)}, \\
& \left\|u^{0}-u^{H}\right\|_{L^{2}(\Omega)} \leq C_{1} H^{\min (p+1, l)}+C_{2} M^{2(1-s)}, \\
& \left\|u^{\varepsilon}-u^{H}\right\|_{L^{2}(\Omega)} \leq C_{1} H^{\min (p+1, l)}+C_{2} M^{2(1-s)}+C_{3} \varepsilon, \\
& \left\|u^{\varepsilon}-u_{p}^{\varepsilon}\right\|_{\bar{H}^{1}(\Omega)} \leq C_{1} H^{\min (p, l)}+C_{2} M^{1-s}+C_{3} \sqrt{\varepsilon},
\end{aligned}
$$

where $C_{2}$ depends on $\left\|a_{i j}\right\|_{L^{\infty}\left(\Omega ; H_{p e r}^{s}(Y)\right)},\left\|\chi^{j}\right\|_{L^{\infty}\left(\Omega ; H_{p e r}^{s}(Y)\right)}$, where $H$ is the size of the triangulation of the macro FE space (2.13), and where $M^{d}$ is the number of pseudospectral points of the microspace $S_{M}\left(K_{\varepsilon}\right)$ given in (2.15).

THEOREM 4.3. Let $u^{0}$ be the solution of the homogenized problem (2.3), and assume $u^{0} \in H^{l+1}(\Omega)$. Let $u^{H}$ be the solution of problem (4.5), and suppose that the assumptions of Lemma 3.6 hold. Then

$$
\begin{aligned}
& \left\|u^{0}-u^{H}\right\|_{H^{1}(\Omega)} \leq C_{1} H^{\min (p, l)}+C_{2}\left(M(\log M)^{(d-1)} e^{-\alpha M}\right)^{2}, \\
& \left\|u^{0}-u^{H}\right\|_{L^{2}(\Omega)} \leq C_{1} H^{\min (p+1, l)}+C_{2}\left(M(\log M)^{(d-1)} e^{-\alpha M}\right)^{2}, \\
& \left\|u^{\varepsilon}-u^{H}\right\|_{L^{2}(\Omega)} \leq C_{1} H^{\min (p+1, l)}+C_{2}\left(M(\log M)^{(d-1)} e^{-\alpha M}\right)^{2}+C_{3} \varepsilon, \\
& \left\|u^{\varepsilon}-u_{p}^{\varepsilon}\right\|_{\bar{H}^{1}(\Omega)} \leq C_{1} H^{\min (p, l)}+C_{2} M(\log M)^{d-1} e^{-\alpha M}+C_{3} \sqrt{\varepsilon},
\end{aligned}
$$

where $C_{2}$ depends on $\mathcal{B}$, where $H$ is the size of the triangulation of the macro $F E$ space (2.13), and where $M^{d}$ is the number of pseudospectral points of the microspace $S_{M}\left(K_{\varepsilon}\right)$ given in (2.15).

5. Numerical experiments. We end this paper with a few numerical experiments illustrating our theory.

5.1. Example 1: Uniformly periodic problem. In order to see the effect of the spectral microsolver and to compare it with a FEM-type microsolver, we consider the (quasi-1-dimensional) model problem (see [3])

$$
\begin{aligned}
& -\nabla \cdot\left(a\left(\frac{x}{\varepsilon}\right) \nabla u^{\varepsilon}\right)=f(x) \quad \text { in } \Omega=(0,1)^{2}, \\
& \left.u^{\varepsilon}\right|_{\Gamma_{D}}=0 \quad \text { on } \Gamma_{D}:=\left\{x_{1}=0\right\} \cup\left\{x_{1}=1\right\} \text {, } \\
& \left.n \cdot\left(a\left(\frac{x}{\varepsilon}\right) \nabla u^{\varepsilon}\right)\right|_{\Gamma_{N}}=0 \quad \text { on } \Gamma_{N}:=\partial \Omega \backslash \Gamma_{D} \text {, }
\end{aligned}
$$

Copyright $@$ by SIAM. Unauthorized reproduction of this article is prohibited. 

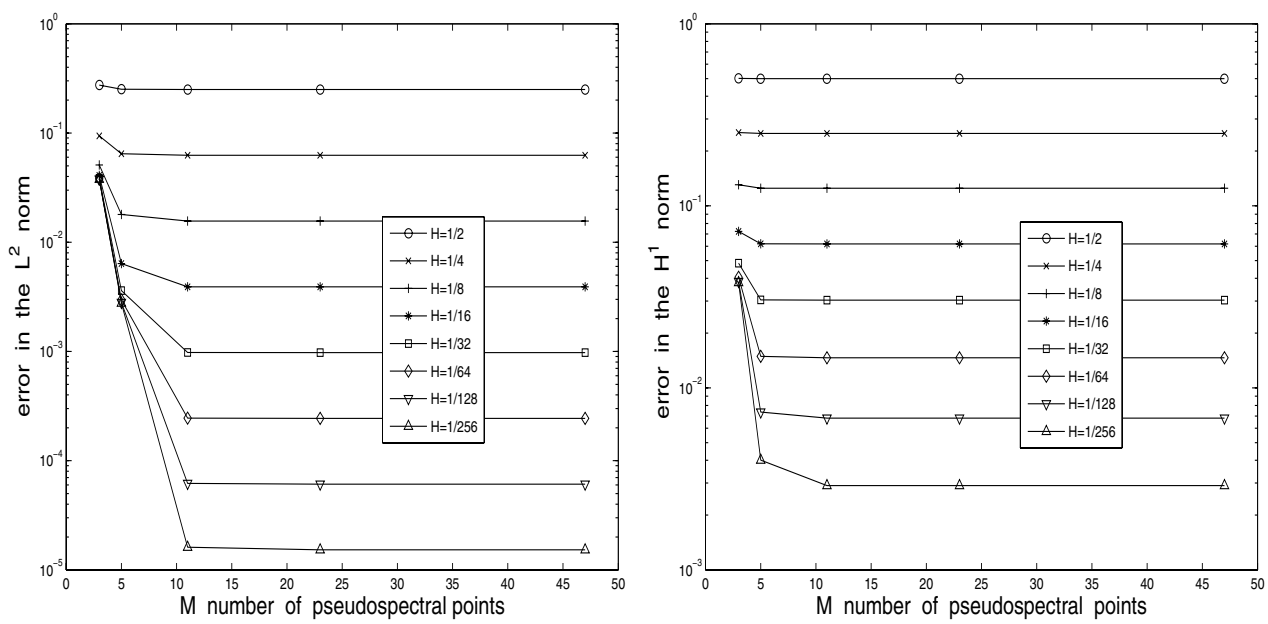

FIG. 1. FES-HMM: micromesh refinement for fixed macromesh $H=2^{-\alpha}, \alpha=1, \ldots, 8$.

where $a(y)=\left(\cos 2 \pi y_{1}+2\right), y=\left(y_{1}, y_{2}\right) \in Y=(0,1)^{2}$, and $f(x) \equiv 1$. The homogenized solution is a quadratic polynomial depending on the homogenized tensor which can be easily computed for this simple problem (see [3] for details). The purpose of the following numerical experiments is to illustrate the different impact of the errors in the microsolver at a macroscale when solving the microproblem with either a FEM or a spectral method. We therefore consider only the homogenized problem in order to be free of the boundary layer term when comparing the fine scale solution with the reconstructed heterogeneous multiscale solution (see section 3.5).

In the figures below, we study the macroconvergence of the FE-HMM (finite element heterogeneous multiscale method with macro- and micropiecewise linear FE spaces) and the FES-HMM (finite element spectral heterogeneous multiscale method with macropiecewise linear FE space and a micropseudospectral method). In both cases, the macromesh is denoted by $H$, and we denote by $h_{L}^{\varepsilon}=h_{L} / \varepsilon$ the meshsize of the micro FEM, where $h_{L}=1 / L$. When using pseudospectral methods $h_{L}^{\varepsilon}=h_{L} / \varepsilon$ will denote the equidistant spacing of the pseudospectral points and $L$ the number of pseudospectral points (in one dimension).

We first study in Figures 1 and 2 for a given macromesh $H$ the influence of the micromesh refinement on the macroerror (for $H$ chosen, $h_{L}=1 / 2,1 / 4,1 / 10,1 / 22$, 1/46). A horizontal line indicates that the microcalculations are precise enough so that the global error is given by the macromesh size (which is kept constant for each experiment). We see for the FES-HMM in Figure 1, both for the $L^{2}$ error (left picture) and the $H^{1}$ error (right picture), that after a short transient $h_{L}<1 / 8$ we have a perfect horizontal line, indicating that the influence of micromesh refinement can no longer be seen. For the FE-HMM, we see in Figure 2 (left) that for the $L^{2}$ norm, the micromesh has to be refined until $h_{L} \simeq H$ in order to stabilize the global error. We see in Figure 2 (right) that for the $H^{1}$ norm, the influence of the microerror is less severe and the micromesh has to be refined until $h_{L} \simeq \sqrt{H}$. These behaviors for the FE-HMM are in accordance with the fully discrete error analysis of [3] (see (2.32) for $l=1$ ), which indicates that both micro- and macromeshes have to be refined simultaneously for the $L^{2}$ norm and that micro- and macromeshes should be refined according to $h_{L} \simeq \sqrt{H}$ for the $H^{1}$ norm. 

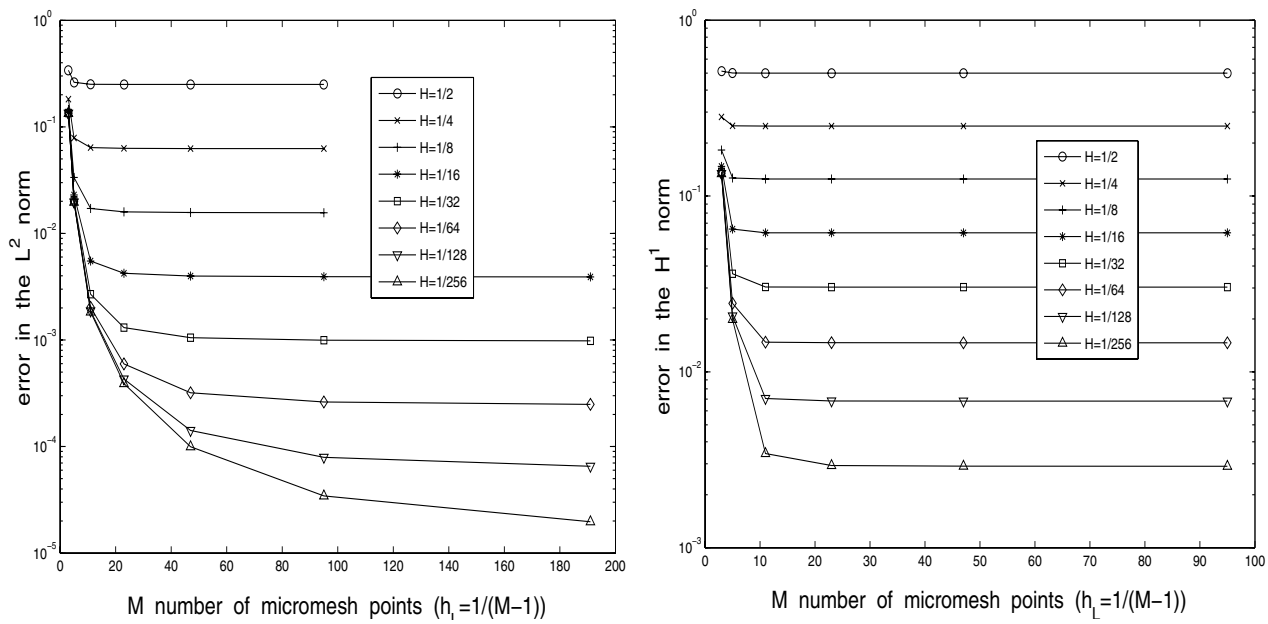

FIG. 2. FE-HMM: micromesh refinement for fixed macromesh $H=2^{-\alpha}, \alpha=1, \ldots, 8$.
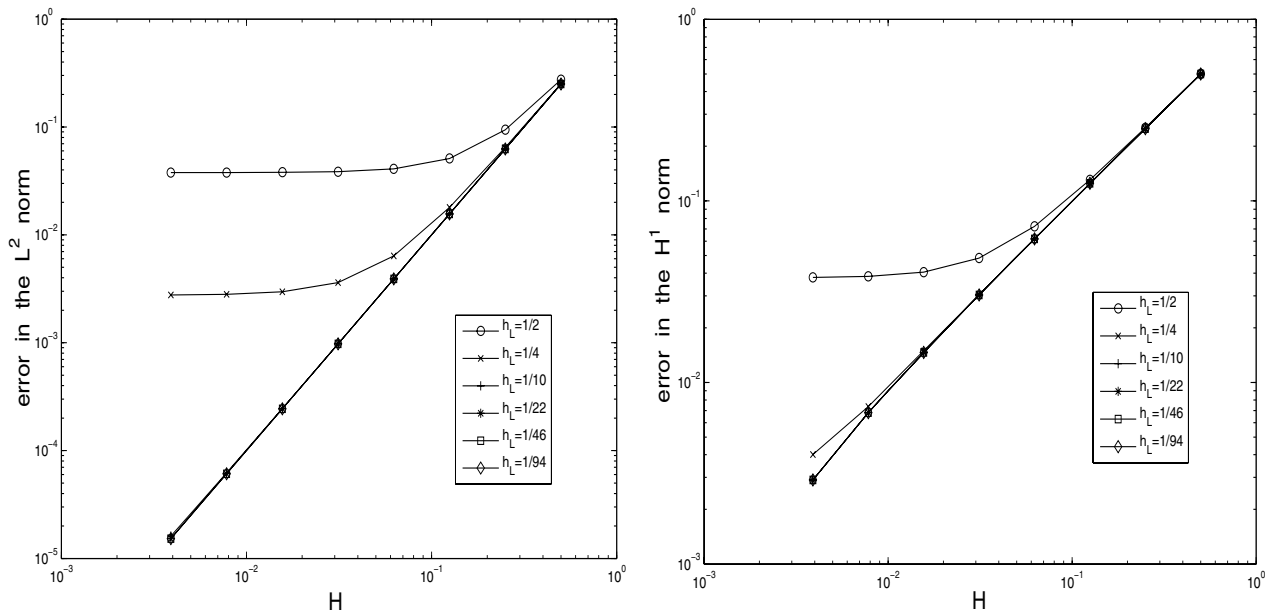

FIG. 3. FES-HMM: macromesh refinement for fixed micromesh $h_{L}=1 / 2,1 / 4,1 / 10,1 / 22,1 / 46$.

We next study macromesh refinements in Figures 3 and 4 . Here we fix the micromesh $h_{L}$ and refine the macromesh $H=2^{-\alpha}, \alpha=1, \ldots, 8$. We see for the FES-HMM in Figure 1, both for the $L^{2}$ error (left picture) and the $H^{1}$ error (right picture) and for all $h_{L} \geq 1 / 8$, that we have perfect quadratic and linear convergence. This again confirms our theoretical results: up to exponential convergence, the error of the FES-HMM is given by the usual error of the macro FEM, i.e., independent of the microsolver. For the FE-HMM, we see in Figure 3 (left) that for the $L^{2}$ norm, the quadratic macroconvergence rate can be observed until $H \simeq h_{L}$. Refining further the macromesh does not decrease the global error any further. For the $H^{1}$ error, the refinement rate $h_{L} \simeq \sqrt{H}$ can be observed. Again, these behaviors for the FE-HMM 

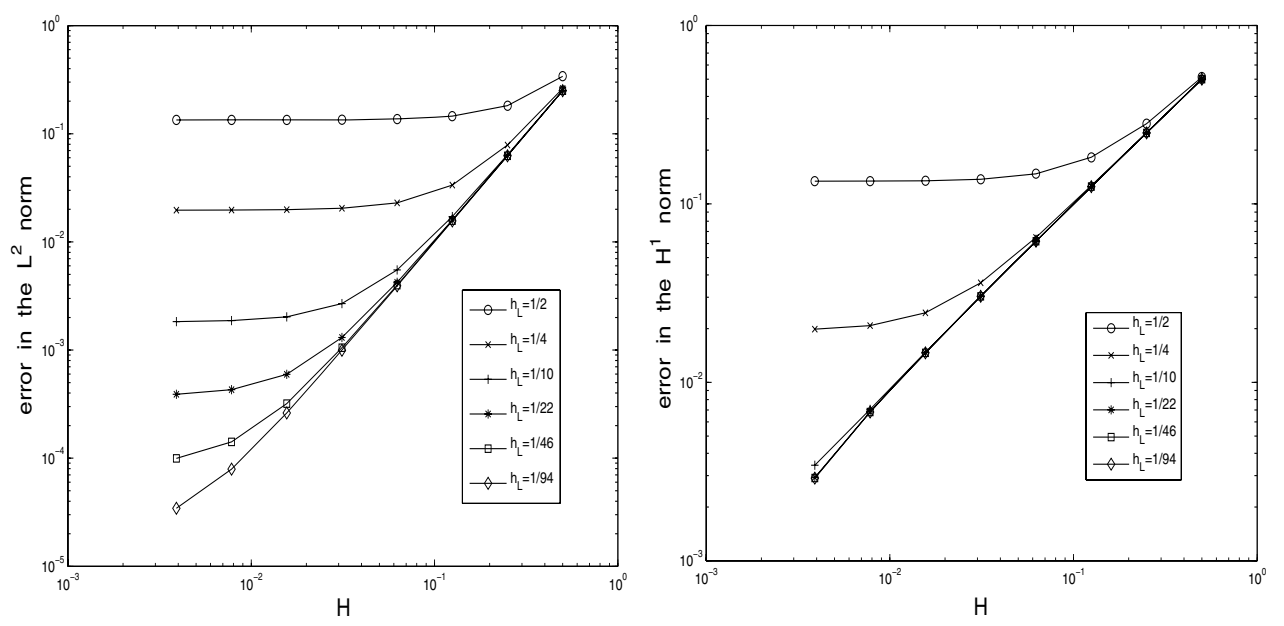

FIG. 4. FE-HMM: macromesh refinement for fixed micromesh $h_{L}=1 / 2,1 / 4,1 / 10,1 / 22,1 / 46$.

are in accordance with the fully discrete error analysis of [3] (see $(2.32)$ for $l=p=1){ }^{2}$

5.2. Example 2: Two-scale problem. We next consider a truly two-scale problem (also considered in [25]) given by

$$
\begin{aligned}
-\nabla \cdot\left(a\left(x, \frac{x}{\varepsilon}\right) \nabla u^{\varepsilon}\right) & =f(x) \quad \text { in } \Omega=(0,1)^{2}, \\
u^{\varepsilon}(x) & =0 \quad \text { on } \partial \Omega,
\end{aligned}
$$

where

$$
a\left(x, \frac{x}{\varepsilon}\right)=\frac{1.5+\sin \left(2 \pi x_{1} / \varepsilon\right)}{1.5+\sin \left(2 \pi x_{2} / \varepsilon\right)}+\frac{1.5+\sin \left(2 \pi x_{2} / \varepsilon\right)}{1.5+\cos \left(2 \pi x_{1} / \varepsilon\right)}+\sin \left(4 x_{1} x 2\right)+1,
$$

where $f=10$ and $x=\left(x_{1}, x_{2}\right)$. This multiscale problem does not have periodic coefficients, but the fast scale is periodic (see Figure 5, right picture). In contrast to the previous example, we do not have an analytical solution for the homogenized problem. We will compute a reference solution of the fine scale problem on a very fine mesh of $10^{6}$ degrees of freedom with a classical FEM. As a measure of convergence (see Corollaries 3.10 and 3.12) study

$$
\frac{\left\|u^{\varepsilon}-u^{H}\right\|_{L^{2}(\Omega)}}{\left\|u^{\varepsilon}\right\|_{L^{2}(\Omega)}} .
$$

We monitor in Figure 5 the convergence results for macromesh refinement $(H=$ $1 / 2,1 / 4,1 / 8,1 / 16 / 1 / 24,1 / 32)$. We study the case when $\delta$ the cell size is equal to the periodicity $\varepsilon$ and the case when the ratio $\delta / \varepsilon=5 / 3$ is noninteger. The number of pseudospectral points is fixed $(M=10$ if $\delta=\varepsilon$ and $M=17$ if $\delta / \varepsilon=5 / 3)$. We

\footnotetext{
${ }^{2}$ Observe that the convergence plots for the $H^{1}$ norm are slightly different from the similar one in [3]. The numerical computation in [3, Figure 4.1, right picture] shows a more severe microerror influence than $h_{L} \simeq \sqrt{H}$, as predicted by the theory given in [3]. This is due to a small error in the code which has been corrected in the present paper. Here the micro-macro refinement $h_{L} \simeq \sqrt{H}$ can be observed.
} 

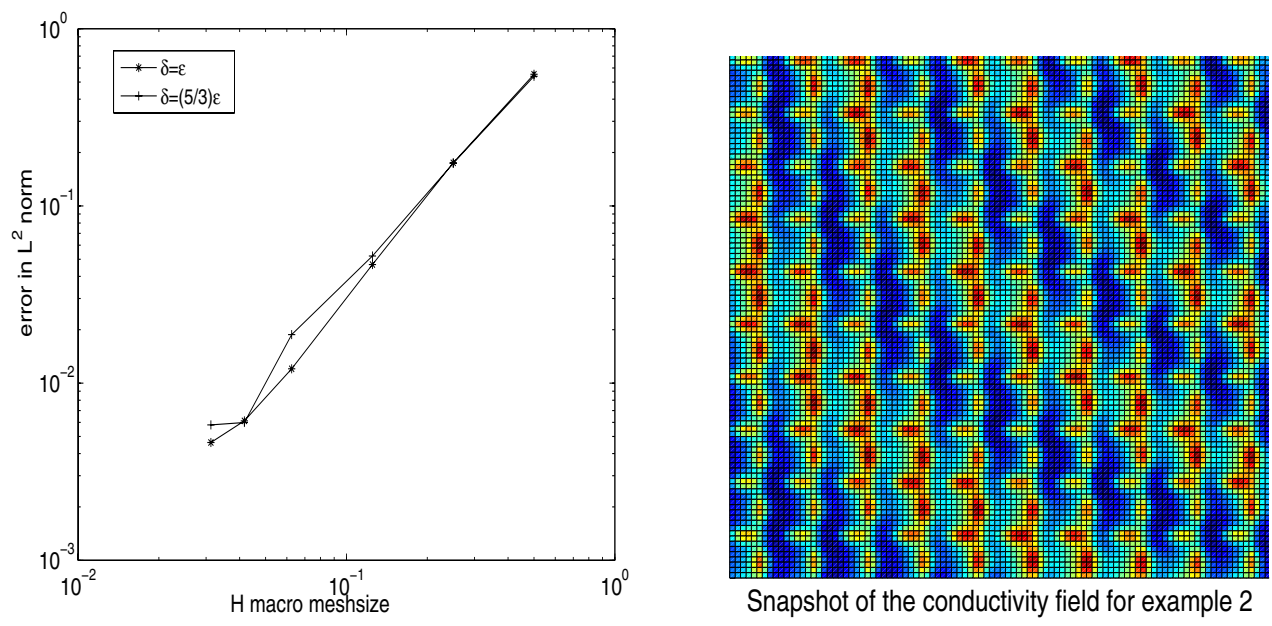

FIG. 5. Convergence of the FES-HMM for example 2 (left picture); snapshot of the conductivity field (right picture).

see that we obtain the expected convergence results independent of $M$ if $\delta=\varepsilon$. For noninteger cell size, the convergence deteriorates at the finest macromesh, but is much better than the a priori estimates one expects for this case, which should involve a boundary layer term $C(\varepsilon / \delta)$ [14]. In general the boundary layer influence for the case of a noninteger ratio between period and cellsize might be larger.

5.3. Example 3: Random porous media problem. For the last example, we consider the multiscale elliptic problem (5.4) with random coefficients $a^{\varepsilon}(x)=$ $a(x / \varepsilon, \omega)$. This is the typical pressure equation for porous media problems. In such a modeling, the natural media is seen as a statistically homogeneous realization of a random field, and the permeability $a^{\varepsilon}(x)$ varies on a $\varepsilon$ length scale much smaller than the characteristic macroscopic length scale of observation. We chose $a^{\varepsilon}$ to be a lognormal stochastic field with mean-zero, variance $\sigma=1$, and correlation length $\varepsilon_{1}=$ $0.02, \varepsilon_{2}=0.03$. We generate a realization of this stochastic field by the moving ellipse averaging method [30]. We then compute a reference solution on a fine $1024 \times 1024$ grid and compare the solution with the FES-HMM (the reference solution can be seen as the exact solution of a finite difference version of (5.4)) [25]. We compute a solution on a coarse $32 \times 32$ grid for the FES-HMM with a sampling domain of size $0.06 \times 0.06$. The error estimates for random coefficients are much weaker (see [14]), and a realistic complexity estimate will require improved analysis.

It can be seen in Figure 6 that the solution obtained from the FES-HMM on a coarse grid $(32 \times 32)$ is in good qualitative agreement with the solution of the standard FEM on the fine grid $(1024 \times 1024$ points $)$.

Appendix A. Spectral estimates. Spectral and exponential convergence results for Fourier pseudospectral methods are usually given for the 1-dimensional case [16], [22], [29], [10]. We briefly discuss in this appendix these approximation results for trigonometric polynomials and extend them, with a tensor product argument, to the multidimensional cases needed for our convergence results.

We consider the notation introduced in section 2.2. Let $u=\sum_{k=-\infty}^{k=\infty} \hat{u}_{k} e^{2 i k \pi y} \in$ $L^{2}(I)$ and $Q_{M}(u)=\sum_{k=-M}^{k=M}{ }^{\prime \prime} \tilde{u}_{k} \psi_{k},|k| \leq M$ the trigonometric interpolant of $u$ at 

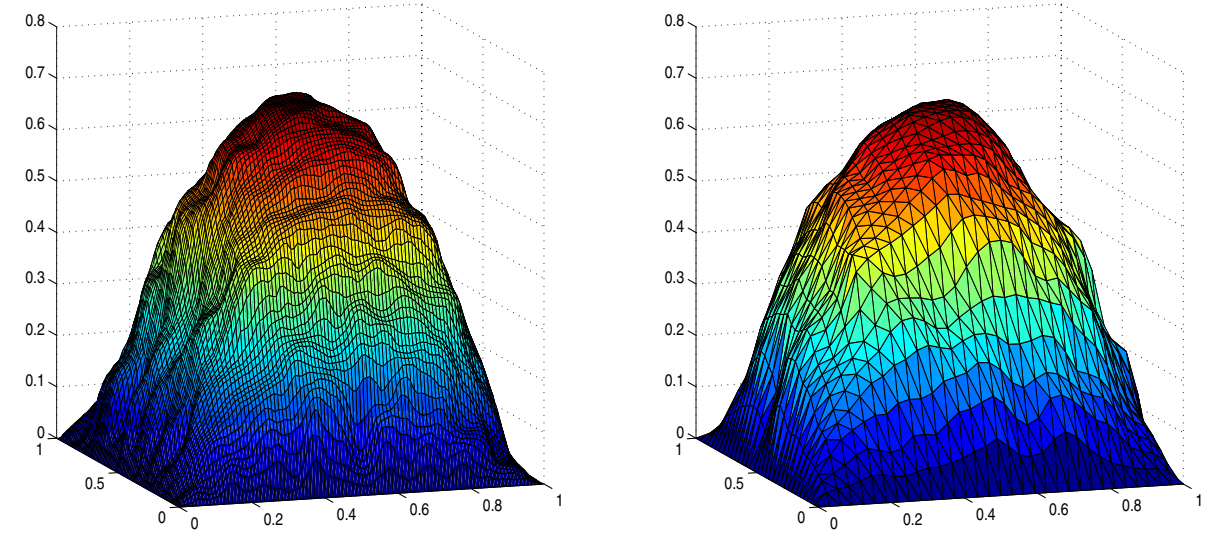

FIG. 6. Comparison of the fine scale solution (pressure profile) of problem (5.4) with random coefficients on a $1024 \times 1024$ grid (left picture) and the FES-HMM solution on a $32 \times 32$ grid (right picture).

the pseudospectral points $I_{M}$ defined in (2.8). In order to estimate the difference $\left\|u-Q_{M}(u)\right\|$ in the $H^{1}$ or in the $L^{2}$ norm, the following lemma, the so-called aliasing lemma, is useful.

Lemma A.1. Assume $u \in C_{p e r}^{0}(I)$; then

$$
\hat{u}_{k}-\tilde{u}_{k}=\sum_{l \neq 0, l=-\infty}^{l=\infty} \hat{u}_{k+2 M l}, \quad-M \leq k \leq M .
$$

This lemma can be proved simply by inserting the Fourier expansion (2.6) into (2.9), and using the orthogonality property

$$
\frac{1}{2 M} \sum_{l=0}^{2 M-1} e^{-2 i \pi k y_{l}}=1 \text { if } k=0(\bmod 2 M) \text { and } 0 \text { otherwise. }
$$

Following [29], using (2.6), (2.9), and (A.1) we can write the difference $u-Q_{M}(u)$ as

$$
u-Q_{M}(u)=-\sum_{k=-M}^{k=M} \prime\left(\sum_{l \neq 0, l=-\infty}^{l=\infty} \hat{u}_{k+2 M l}\right) e^{2 i \pi k x}+\sum_{|k| \geq M}^{\prime \prime} \hat{u}_{k} e^{2 i \pi k x} .
$$

The next lemma gives the so-called spectral accuracy estimates for $\left\|u-Q_{M}(u)\right\|$ and is obtained by estimating both terms in the right-hand side of (A.3) (see, for example, [22] or [29] for details) [10, p. 279].

Lemma A.2. Assume $u \in H_{\text {per }}^{s}(I)$ with $s>1 / 2$; then for any real $\sigma 0 \leq \sigma \leq s$

$$
\left\|u-Q_{M}(u)\right\|_{H^{\sigma}(I)} \leq C M^{\sigma-s}\|u\|_{H^{s}(I)} .
$$

Using (A.4) with $\sigma=s=1$ gives the following stability result:

$$
\left\|\nabla Q_{M}(u)\right\|_{L^{2}(I)} \leq C\|u\|_{H^{1}(I)} .
$$


For the estimates used in section 3 we need the following lemma, proved in [29], which gives an exponential decay estimate, provided $u$ is analytic (see Definition 3.4).

Lemma A.3. Suppose $u \in \mathcal{A}_{B, \alpha}(I)$; then

$$
\left\|u-Q_{M}(u)\right\|_{H^{\sigma}(I)} \leq C B(\alpha) M^{\sigma} e^{-\alpha M},
$$

where $B(\alpha)=\max _{|\Im z| \leq \alpha}|u(z)|$ and the constant $C$ depends on $\alpha$ and $\sigma$.

We will also need the $L^{\infty}$-stability estimate

$$
\left\|Q_{M}(u)\right\|_{L^{\infty}(I)} \leq C \log M\|u\|_{L^{\infty}(I)}
$$

which is proved in [20, pp. 119-121], and the result of Bernstein [8] (see also [24, pp. 56-57]):

$$
\left\|\nabla Q_{M}(u)\right\|_{L^{\infty}(I)} \leq M\left\|Q_{M}(u)\right\|_{L^{\infty}(I)} .
$$

In section 3 we used the $d$-dimensional version of the above approximation results in $Y=I^{d}=(0,1)^{d}$. We recall the tensor product construction given in (2.11) and the notation $\mathbf{Q}_{M}(u)=Q_{M}^{1} \cdots Q_{M}^{d}(u)$ introduced in section 2.2. For simplicity of notation we present the proof for the case $d=2$ and note $\mathbf{Q}_{M}(u)=Q_{M}^{x} Q_{M}^{y}(u)$. We emphasize that the same proofs work in higher dimension. We will also use the isomorphism $L^{2}(Y)=L^{2}\left(I ; L^{2}(I)\right)$, with the usual definition of the latter space.

LEMmA A.4. Let $Y=(0,1)^{d}, d=2,3$, and assume $u \in H_{\text {per }}^{s}(Y)$ with $s \geq 2$; then

$$
\begin{aligned}
& \left\|u-\mathbf{Q}_{M}(u)\right\|_{L^{2}(Y)} \leq C M^{-s}\|u\|_{H^{s}(Y)}, \\
& \left\|\nabla u-\nabla \mathbf{Q}_{M}(u)\right\|_{L^{2}(Y)} \leq C M^{1-s}\|u\|_{H^{s}(Y)} .
\end{aligned}
$$

Proof. Following [7], we write (for $d=2)\left\|u-Q_{M}^{x} Q_{M}^{y}(u)\right\|_{L^{2}(Y)}$

$$
\begin{aligned}
& \leq\left\|u-Q_{M}^{x}(u)\right\|_{L^{2}\left(I ; L^{2}(I)\right)}+\left\|u-Q_{M}^{y}(u)\right\|_{L^{2}\left(I ; L^{2}(I)\right)}+\left\|\left(I d-Q_{M}^{x}\right)\left(I d-Q_{M}^{y}\right) u\right\|_{L^{2}\left(I ; L^{2}(I)\right)} \\
& \leq C M^{-s}\|u\|_{H^{s}\left(I ; L^{2}(I)\right)}+C M^{-s}\|u\|_{L^{2}\left(I ; H^{s}(I)\right)}+C M^{-1} M^{-(s-1)}\|u\|_{H^{1}\left(I, H^{s-1}(I)\right)},
\end{aligned}
$$

where we used (A.4) for the $x$ and $y$ variables in the first and second terms of the right-hand side of the inequality, and successively (A.4) for the $x$ variable and for the $y$ variable. Using the continuous embedding $H^{s}(Y) \subset H^{1}\left(I, H^{s-1}(I)\right)$ concludes the proof of (A.9). To prove (A.10) we first estimate $\left\|\partial_{x} u-\partial_{x} Q_{M}^{x} Q_{M}^{y}(u)\right\|_{L^{2}(Y)}^{2}$

$$
\begin{aligned}
& \leq\left\|\partial_{x} u-\partial_{x} Q_{M}^{x}(u)\right\|_{L^{2}\left(I ; L^{2}(I)\right)}^{2}+\left\|\partial_{x} Q_{M}^{x}\left(u-Q_{M}^{y}(u)\right)\right\|_{L^{2}\left(I ; L^{2}(I)\right)}^{2} \\
& \leq C M^{2(1-s)}\|u\|_{H^{s}\left(I ; L^{2}(I)\right)}^{2}+C\left(\left\|\partial_{x}\left(u-Q_{M}^{y}(u)\right)\right\|_{L^{2}\left(I ; L^{2}(I)\right)}^{2}+\left\|u-Q_{M}^{y}(u)\right\|_{L^{2}\left(I ; L^{2}(I)\right)}^{2}\right),
\end{aligned}
$$

where we used (A.4) and the stability result (A.5). In the second term of the last inequality, we observe that $\partial_{x}$ and $Q_{M}^{y}$ commute. We can then use (A.4) for both the second and the last term and bound them by $C M^{2(1-s)}\|u\|_{H^{s}(Y)}^{2}$. Using a similar argument for $\left\|\partial_{y} u-\partial_{y} Q_{M}^{x} Q_{M}^{y}(u)\right\|_{L^{2}(Y)}^{2}$, summing up and taking the square root yields (A.10).

Lemma A.5. Let $Y=(0,1)^{d}$, and suppose $u \in \mathcal{A}_{\mathcal{B}, \alpha}(Y)$; then

$$
\begin{aligned}
& \left\|u-\mathbf{Q}_{M}(u)\right\|_{L^{2}(Y)} \leq C \mathcal{B} \log M^{d-1} e^{-\alpha M}, \\
& \left\|\nabla u-\nabla \mathbf{Q}_{M}(u)\right\|_{L^{2}(Y)} \leq C \mathcal{B} M \log M^{d-1} e^{-\alpha M},
\end{aligned}
$$

Copyright (C) by SIAM. Unauthorized reproduction of this article is prohibited. 
where $\mathcal{B}=\max _{i \leq d}\left\{\max _{Y_{-i}} \max _{z \in \mathcal{E}_{\alpha}}\left|u\left(Y_{-i}, z\right)\right|\right\}$ (see Definition 3.4) and the constant $C$ depends on $\alpha$.

Proof. For (A.11), we have (for $d=2)\left\|u-Q_{M}^{x} Q_{M}^{y}(u)\right\|_{L^{2}(Y)}$

$\leq\left\|u-Q_{M}^{x}(u)\right\|_{L^{2}\left(I ; L^{2}(I)\right)}+\left\|u-Q_{M}^{y}(u)\right\|_{L^{2}\left(I ; L^{2}(I)\right)}+\left\|\left(I d-Q_{M}^{x}\right)\left(I d-Q_{M}^{y}\right) u\right\|_{L^{2}\left(I ; L^{2}(I)\right)}$.

For the first term of the right-hand side of the inequality, we use (A.6) and (A.7) and bound it by

$$
\begin{aligned}
& \left\|u-Q_{M}^{x}(u)\right\|_{L^{2}\left(I ; L^{2}(I)\right)}^{2}=\int_{I} \int_{I}\left|u(x, y)-Q_{M}^{x}(u)(x, y)\right|^{2} d x d y \\
& \leq C e^{-2 \alpha M} \max _{z \in \mathcal{E}_{\alpha}} \int_{I}|u(z, y)|^{2} d y \leq C e^{-2 \alpha M} \max _{z \in \mathcal{E}_{\alpha}} \max _{y \in I}|u(z, y)|^{2} \leq C \mathcal{B}^{2} e^{-2 \alpha M} .
\end{aligned}
$$

The second term is treated similarly. For the last term we use (A.6) and (A.7) and bound it by

$$
\begin{aligned}
& \left\|\left(I d-Q_{M}^{x}\right)\left(I d-Q_{M}^{y}\right) u\right\|_{L^{2}\left(I ; L^{2}(I)\right)}^{2} \leq C e^{-2 \alpha M} \max _{z \in \mathcal{E}_{\alpha}} \int_{I}\left|u(z, y)-Q_{M}^{y}(u)(z, y)\right|^{2} d y \\
& \leq C e^{-2 \alpha M} \max _{z \in \mathcal{E}_{\alpha}} \max _{y \in I}\left|u(z, y)-Q_{M}^{y}(u)(z, y)\right|^{2} \leq C \mathcal{B}^{2}(\log M)^{2} e^{-2 \alpha M} .
\end{aligned}
$$

Summing and taking the square root yields (A.11).

To prove (A.12) we first estimate $\left\|\partial_{x} u-\partial_{x} Q_{M}^{x} Q_{M}^{y}(u)\right\|_{L^{2}(Y)}^{2}$

$$
\leq\left\|\partial_{x} u-\partial_{x} Q_{M}^{x}(u)\right\|_{L^{2}\left(I ; L^{2}(I)\right)}^{2}+\left\|\partial_{x} Q_{M}^{x}\left(u-Q_{M}^{y}(u)\right)\right\|_{L^{2}\left(I ; L^{2}(I)\right)}^{2} .
$$

For the first term of the right-hand side, we use (A.6) and bound it by $C \mathcal{B}^{2} M^{2} e^{-2 \alpha M}$. For the second term of the right-hand side, we first use (A.8) and then (A.7), and we obtain the bound $C \mathcal{B}^{2} M^{2} \log M^{2} e^{-2 \alpha M}$. Using a similar argument for $\| \partial_{y} u-$ $\partial_{y} Q_{M}^{x} Q_{M}^{y}(u) \|_{L^{2}(Y)}^{2}$, summing up and taking the square root yields (A.12).

Acknowledgment. The authors are grateful to Christoph Schwab for helpful discussions.

\section{REFERENCES}

[1] A. Abdulle And W. E, Finite difference HMM for homogenization problems, J. Comput. Phys., 191 (2003), pp. 18-39.

[2] A. Abdulle and C. Schwab, Heterogeneous multiscale FEM for diffusion problems on rough surfaces, Multiscale Model. Simul., 3 (2005), pp. 195-220.

[3] A. ABDULle, On a priori error analysis of fully discrete heterogeneous multiscale FEM, Multiscale Model. Simul., 4 (2005), pp. 447-459.

[4] A. Abdulle, Multiscale methods for advection-diffusion problems, Discrete Contin. Dyn. Syst., Suppl. Vol. (2005), pp. 11-21.

[5] A. AbDulle, Analysis of a heterogeneous multiscale FEM for problems in elasticity, Math. Models Methods Appl. Sci., 16 (2006), pp. 615-635.

[6] A. Bensoussan, J.-L. Lions, and G. Papanicolaou, Asymptotic Analysis for Periodic Structures, North-Holland, Amsterdam, 1978.

[7] C. Bernardi, Y. Maday, and F. Rapetti, Discrétisations variationnelles de problèmes aux limites elliptiques, Math. Appl. (Berlin) 45, Springer, Berlin, 2004.

[8] S. N. Bernstein, Sur l'ordre de la meilleure approximation des fonctions continues par les polynômes de degré donné, Mémoire de l'Acad. Royale de Belgique, 4 (1912), pp. 1-104.

[9] L. Bers, F. John, and M. Schechter, Partial Differential Equations, Lectures in Applied Mathematics, Proceedings of the Summer Seminar, Boulder, CO, 1957.

Copyright (c) by SIAM. Unauthorized reproduction of this article is prohibited. 
[10] C. Canuto, M. Y. Hussaini, A. Quarteroni, and T. A. Zang, Spectral Methods in Fluid Dynamics, Springer, New York, 1987.

[11] P. G. Ciarlet, The Finite Element Method for Elliptic Problems, North-Holland, Amsterdam, 1978.

[12] D. Cioranescu and P. Donato, An Introduction to Homogenization, Oxford University Press, New York, 1999.

[13] W. E And B. Engquist, The heterogeneous multiscale methods, Commun. Math. Sci., 1 (2003), pp. $87-132$.

[14] W. E, P. Ming, And P. Zhang, Analysis of the heterogeneous multi-scale method for elliptic homogenization problems, J. Amer. Math. Soc., 18 (2004), pp. 121-156.

[15] B. EngQuist And Y.-H. Tsai, Heterogeneous multiscale methods for stiff ordinary differential equations, Math. Comp., 74 (2005), pp. 1707-1742.

[16] D. Gottlieb and S. A. Orszag, Numerical Analysis of Spectral Methods: Theory and Applications, CBMS-NSF Regional Conf. Ser. in Appl. Math. 26, SIAM, Philadelphia, 1977.

[17] V. H. HoAng AND S. Schwab, High-dimensional finite elements for elliptic problems with multiple scales, Multiscale Model. Simul., 3 (2005), pp. 168-194.

[18] T.-Y. Hou, X.-H. Wu, AND Z. CAI, Convergence of a multiscale finite element method for elliptic problems with rapidly oscillating coefficients, Math. Comp., 68 (1999), pp. 913943.

[19] T. Hou And X.-H. Wu, A multiscale finite element method for elliptic problems in composite materials and porous media, J. Comput. Phys., 134 (1997), pp. 169-189.

[20] D. Jackson, The Theory of Approximation, Amer. Math. Soc. Colloq. Publ. 11, AMS, New York, 1930.

[21] V. V. Jikov, S. M. Kozlov, And O. A. Oleinik, Homogenization of Differential Operators and Integral Functionals, Springer, Berlin, Heidelberg, 1994.

[22] H.-O. Kreiss and J. Oliger, Stability of the Fourier method, SIAM J. Numer. Anal., 16 (1979), pp. 421-433.

[23] O. A. Ladyzhenskaya and N. N. Ural'tseva, Linear and Quasilinear Elliptic Equations, Academic Press, New York, 1968.

[24] G. Meinardus, Approximation von Funktionen und ihre numerische Behandlung, Springer Tracts Nat. Philos. 4, Springer, Berlin, New York, 1964.

[25] P. Ming And Y. Yue, Numerical methods for multiscale elliptic problems, J. Comput. Phys., 214 (2006), pp. 421-445.

[26] S. Moskow And M. Vogelius, First order corrections to the homogenised eigenvalues of a periodic composite medium. A convergence proof, Proc. Roy Soc. Edinburgh Sect. A, 127 (1997), pp. 1263-1299.

[27] L. Nirenberg AND C. B. Morrey, JR., On the analycity of the solution of linear elliptic systems of partial differential equations, Comm. Pure Appl. Math., 10 (1957), pp. 271290.

[28] J. T. Oden And K. S. Vemaganti, Estimation of local modeling error and global-oriented adaptive modeling of heterogeneous materials: Error estimates and adaptive algorithms, J. Comput. Phys., 164 (2000), pp. 22-47.

[29] E. TADMOR, The exponential accuracy of Fourier and Chebyshev differencing methods, SIAM J. Numer. Anal., 23 (1986), pp. 1-10.

[30] T. C. Wallstrom, S. Hou, M. A. Christie, L. J. Durlofsky, and D. H. Sharp, Accurate scale up of two phase flow using renormalization and nonuniform coarsening, Comput. Geosci., 3 (1999), pp. 69-87.

Copyright (c) by SIAM. Unauthorized reproduction of this article is prohibited. 